ARTICLE

DOI: $10.1038 / s 41467-018-04592-z$

\title{
Spatio-temporal relays control layer identity of direction-selective neuron subtypes in Drosophila
}

Holger Apitz (iD ${ }^{1}$ \& Iris Salecker (10 ${ }^{1}$

\begin{abstract}
Visual motion detection in sighted animals is essential to guide behavioral actions ensuring their survival. In Drosophila, motion direction is first detected by T4/T5 neurons. Their axons innervate one of the four lobula plate layers. How T4/T5 neurons with layer-specific representation of motion-direction preferences are specified during development is unknown. We show that diffusible Wingless $(\mathrm{Wg}$ ) between adjacent neuroepithelia induces its own expression to form secondary signaling centers. These activate Decapentaplegic (Dpp) signaling in adjacent lateral tertiary neuroepithelial domains dedicated to producing layer 3/4-specific T4/T5 neurons. T4/T5 neurons derived from the core domain devoid of Dpp signaling adopt the default layer $1 / 2$ fate. Dpp signaling induces the expression of the T-box transcription factor Optomotor-blind (Omb), serving as a relay to postmitotic neurons. Omb-mediated repression of Dachshund transforms layer 1/2- into layer 3/4-specific neurons. Hence, spatio-temporal relay mechanisms, bridging the distances between neuroepithelial domains and their postmitotic progeny, implement T4/T5 neuron-subtype identity.
\end{abstract}

\footnotetext{
${ }^{1}$ The Francis Crick Institute, Visual Circuit Assembly Laboratory, 1 Midland Road, London NW1 1AT, UK. Correspondence and requests for materials should be addressed to I.S. (email: iris.salecker@crick.ac.uk)
} 
$\mathrm{V}$ isual signals received by the retina are generally not stationary because objects in the environment and/or the bodies of animals move. To detect motion, visual circuits perform complex spatio-temporal comparisons that convert luminance changes collected by photoreceptors into signals containing information about direction or speed. Despite the seemingly divergent anatomy of vertebrate and insect visual systems, they display remarkable parallels in the computations underlying motion vision and the neuronal elements performing them ${ }^{1,2}$. In most sighted animals, this involves neurons that respond to motion signals in specific directions. Directionselectivity emerges from differences in the connectivity of their dendrites $^{2}$. Motion-direction preferences by their axons are represented by layer-specific innervation ${ }^{3-8}$. Thus, anatomical characteristics such as layer-specificity seem to be intricately linked with motion-directionality. However, how these are implemented during circuit development is poorly understood.

The Drosophila visual system has emerged as a powerful model for elucidating the neural circuits and computations underlying motion detection. Photoreceptors (R-cells) in the retina extend axons into the optic lobe consisting of the lamina, medulla, lobula plate, and lobula (Fig. 1a). Neuronal projections in these ganglia are organized into retinotopically arranged columnar units. The medulla, lobula plate, and lobula are additionally subdivided into synaptic layers. They are innervated by more than a 100 neuronal subtypes that extract different visual features in parallel pathways ${ }^{9}$. T4 and T5 lobula plate neurons are the first directionselective circuit elements ${ }^{6,10}$. Each optic lobe hemisphere contains $\sim 5300$ T4/T5 neurons ${ }^{11}$. T4 dendrites arborize within medulla layer 10, and T5 dendrites in lobula layer Lo1. Their axons project to one of the four lobula plate layers, thereby defining four different neuron subtypes each ${ }^{12}$ (Fig. 1a). Axons segregate according to their motion-direction preferences. Thus, front-toback, back-to-front, upward, and downward cardinal motion directions are represented in lobula plate layers $1-4^{5,6}$. T4 neurons are part of the $\mathrm{ON}$ motion detection pathway reporting brightness increments, while T5 neurons are part of the OFF pathway reporting brightness decrements ${ }^{6}$. Distinct neuron sets in the lamina and medulla relay $\mathrm{ON}$ and $\mathrm{OFF}$ information to $\mathrm{T} 4$ and T5 neurons ${ }^{2,13}$. Direction-selectivity emerges within T4/T5 dendrites and involves the non-linear integration of input from these upstream neurons for enhancement in the preferred direction and suppression in the null-direction ${ }^{10,14-16}$. Dendritic arbors of the four T4 neuron subtypes have characteristic orientations, that correlate with the direction preferences of lobula plate layers innervated by their axons ${ }^{17,18}$. Thus, directionselectivity involves the establishment of neuron subtypes, each with distinct spatial connectivities. Here, we address when and how T4 and T5 neuron subtypes with different layer identities are specified during development.

Optic lobe neurons originate from two horseshoe-shaped neuroepithelia, called the outer and inner proliferation centers (OPC and IPC; Fig. 1b) ${ }^{19,20}$. These are derived from the embryonic optic lobe placode ${ }^{21}$ and expand by symmetric cell divisions during early larval development ${ }^{22,23}$. At the late 2 nd instar larval stage, neuroepithelial (NE) cells from the medial OPC edge begin to transform into medulla neural stem cells, called neuroblasts $(\mathrm{Nbs})^{20}$. These undergo asymmetric divisions to self-renew and give rise to ganglion mother cells (GMCs), which divide to generate two neurons or glia ${ }^{22,24}$. Apposing the OPC, two dorsal and ventral NE domains, called the glial precursor cell (GPC) areas, produce neuron subtypes associated with all ganglia ${ }^{25,26}$. At the mid 3rd instar larval stage, the lateral OPC begins to generate lamina neurons ${ }^{20}$.

The IPC generates lobula and lobula plate neurons, including T4/T5 neurons from the early 3rd instar larval stage onward $^{20}$. Our recent studies showed that $\mathrm{NE}$ cells in one domain, the proximal (p-)IPC, convert into progenitors in an epithelial-mesenchymal transition (EMT)-like process ${ }^{23,27}$. Progenitors migrate to a second proliferative zone, the distal (d-)IPC, where they mature into Nbs. These transition through two competence windows to first produce C\&T neurons, corresponding to $\mathrm{C} 2$ and $\mathrm{C} 3$ ascending neurons connecting the medulla and lamina, as well as T2/T2a and T3 neurons connecting the medulla and lobula ${ }^{12}$, and then T4/T5 lobula plate neurons (Fig. 1a, b). Cross-regulatory interactions between Dichaete (D) and Tailless (Tll) control the switch in Nb competence defined by the sequential expression of the proneural bHLH transcription factors Asense (Ase) and Atonal (Ato). The latter is co-expressed with the retinal determination protein Dachshund $(\mathrm{Dac})^{23}$. The molecular mechanisms that control layer-specific T4/T5 neuron subtype identities within this sequence of developmental events occurring at different locations have remained elusive.

T4/T5 neuron diversity resulting in differential layer-specificity could be achieved by postmitotic combinatorial transcription factor codes upstream of distinct guidance molecules. Although not mutually exclusive, layer-specificity of T4/T5 neurons could also be determined by temporal differences in the expression of common postmitotic determinants, similar to the birth-order dependent R-cell growth cone segregation strategy described in the medulla ${ }^{28,29}$. Here, we provide evidence for another mechanism, whereby layer-specific T4/T5 neuron subtype identity is determined early in the p-IPC neuroepithelium. Their specification depends on two relay mechanisms involving Wnt and Bone morphogenetic protein (Bmp) signaling and transcription factor interactions. These establish and translate the spatial patterning of NE cells into postmitotic neuronal subtype identities to bridge distances inherent to this particular neurogenesis mode.

\section{Results}

Layer 3/4 innervating T4/T5 neurons depend on Wg secretion. Wnt family members are evolutionary conserved signaling proteins that orchestrate tissue patterning and growth during development. Recent findings showed that flies expressing membrane-tethered instead of normally secreted Wingless (Wg) were viable and had normal bodies and well-patterned, albeit slightly smaller wings ${ }^{30}$. This indicated that long-range spreading of $\mathrm{Wg}$ is not essential for the development of many tissues. As the general fitness of these flies was reduced, Wg release could possibly be required in tissues other than imaginal discs. In the visual system, Wg is expressed and required in the GPC areas for neuron specification $25,26,31,32$. To explore whether Wg plays a role in the IPC and the spreading of this signaling molecule is essential, we examined the brains of adult homozygous flies, engineered to solely express Wg fused to the type-2 transmembrane protein Neurotactin ${ }^{30}$ (wg $\{K O ; N R T-w g\} ;$ Fig. 1c). We uncovered a specific and highly penetrant phenotype in the lobula plate (Fig. $1 \mathrm{~d}-\mathrm{g})$ : unlike in controls $(n=13 / 13)$, either one $(n=$ $18 / 30)$ or two $(n=12 / 30)$ of the four lobula plate layers were missing. Moreover, T4/T5 neuron numbers were reduced by approximately $25 \%$ in three-layered and $50 \%$ in two-layered samples, respectively (Fig. 1h). To determine which layers were affected, optic lobes were immunolabeled with the cell surface molecule Connectin, a specific marker of lobula plate layers $3 / 4$. In contrast to controls $(n=26 / 26)$, Connectin was expressed either in one $(n=16 / 30)$ or none $(n=14 / 30)$ of the lobula plate layers in $w g\{K O ; N R T-w g\}$ flies (Fig. 1i-k). Thus, T4/T5 neurons innervating layers $3 / 4$ were preferentially affected in flies solely expressing membrane-tethered $\mathrm{Wg}$. 
To elucidate the underlying causes, we examined the expression of wild-type $\mathrm{Wg}$ in the 3rd instar larval optic lobe. Consistent with previous reports ${ }^{26}, \mathrm{Wg}$ protein was detected in the dorsal and ventral GPC areas adjacent to the OPC (Fig. 1l, n, o). Apposing the ventral p-IPC shank, Wg was expressed in surface (s-)IPC NE cells, which generate two lobula neuron clusters ${ }^{23}$. Additionally, $\mathrm{Wg}$ was expressed in a small $\mathrm{Nb}$ clone adjacent to the dorsal p-IPC shank (Fig. 1m-o). Relying on the perdurance of reporter gene expression by two $w g$-Gal4 drivers to mark the progeny of these domains a
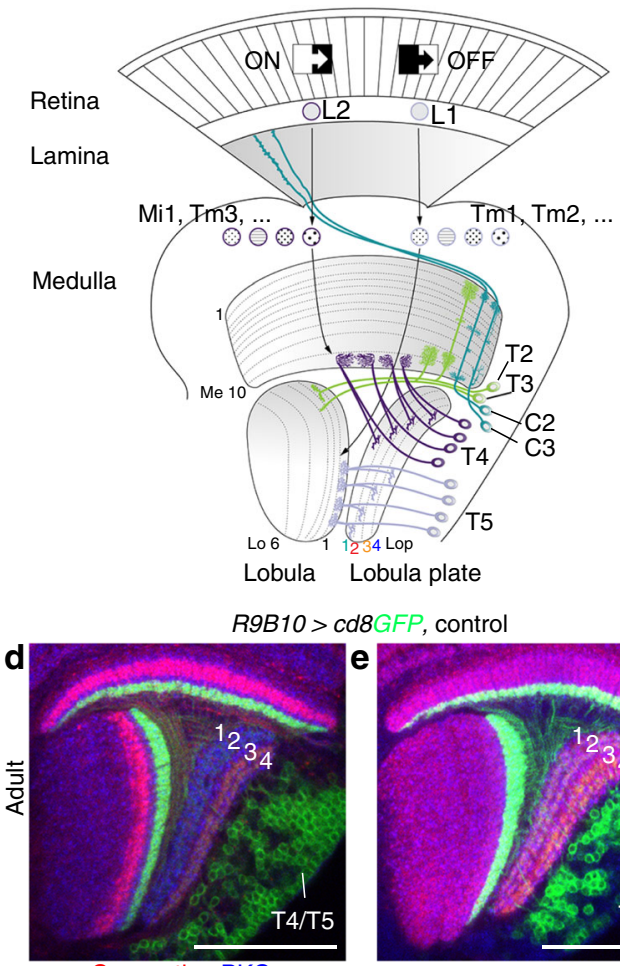

Connectin aPKC
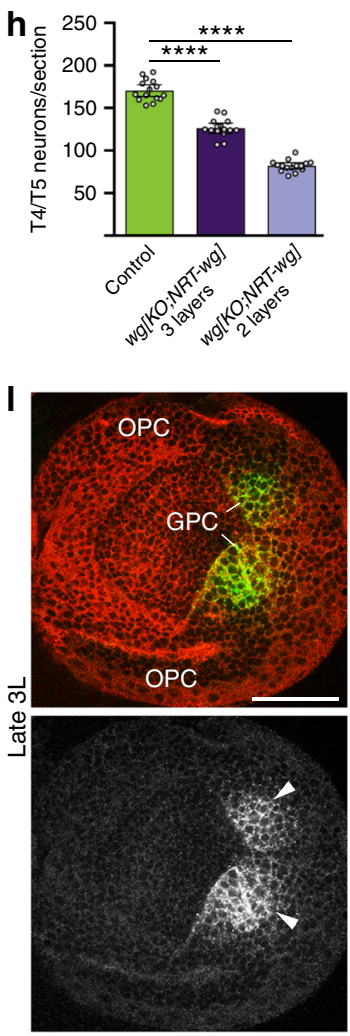

Lateral view

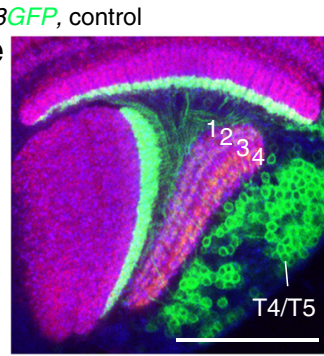

Control

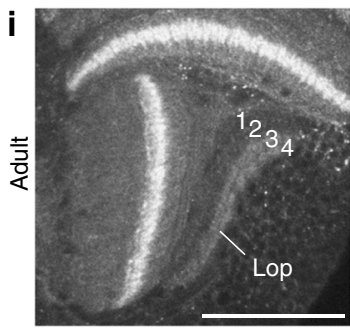

Wild type

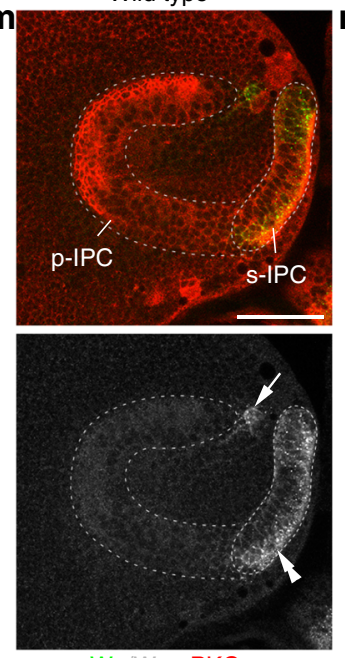

$\mathrm{Wg} / \mathrm{Wg}$ aPKC b

Lamina

Medulla

Lateral

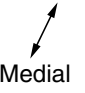

C

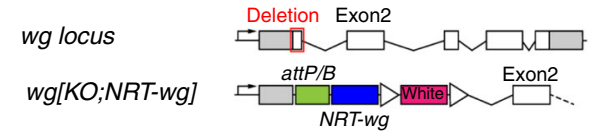

$R 9 B 10>c d 8 G F P, w g[K O ; N R T-w g]$

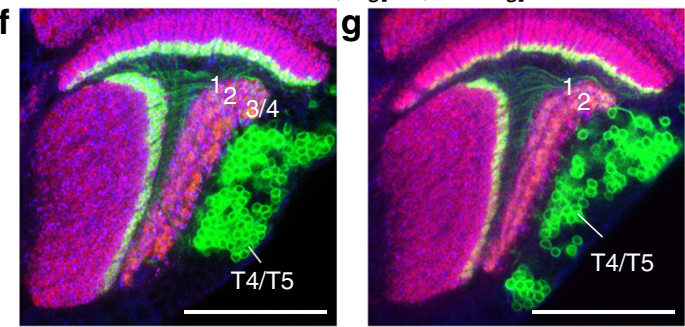

Brp aPKC

$w g[K O ; N R T-w g]$
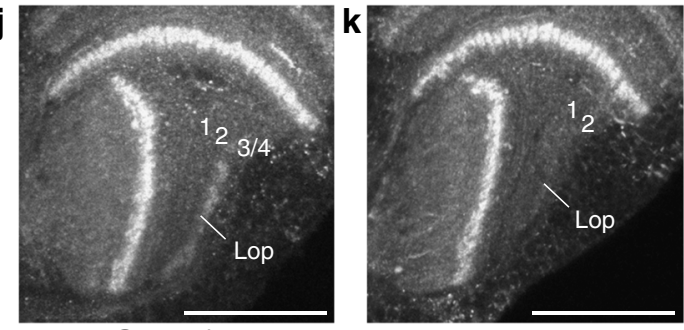

Connectin
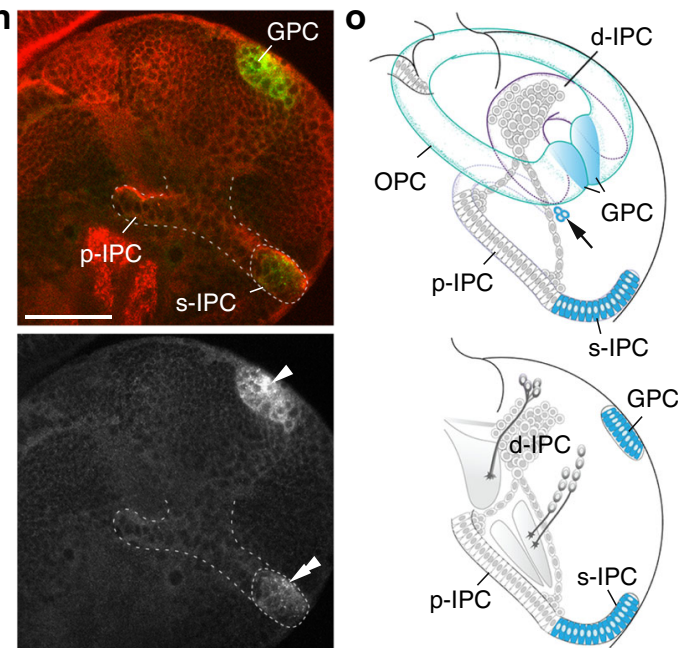

Horizontal view

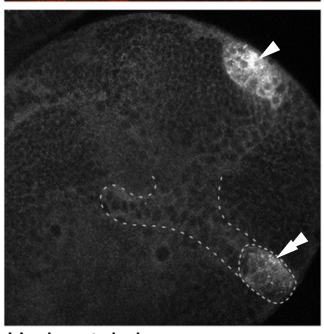

- Wg 
(Supplementary Fig. 1a-d), we observed that $w g$ is not produced in T4/T5 neurons or their progenitors in the d-IPC and p-IPC. This suggests a non-autonomous requirement of $\mathrm{Wg}$ for the development or survival of T4/T5 neurons innervating layers $3 / 4$.

Wg secretion from the GPC areas induces $w g$ in secondary domains. We next asked how the prevention of $\mathrm{Wg}$ release might cause the lobula plate-specific defects. In the GPC areas, Wg blocks the expression of the transcriptional regulator Homothorax (Hth), a temporal series factor in the $\mathrm{OPC}^{32}$. Hth expression was correctly suppressed in $w g\{K O ; N R T-w g\}$ flies, suggesting that NRT-Wg functions as wild-type Wg in this context (Supplementary Fig. 2a, b). However in the IPC (Fig. 2a, b; Supplementary Fig. 2c-f), NRT-Wg expression was absent in the s-IPC adjacent to the ventral p-IPC and the $\mathrm{Nb}$ clone adjacent to the dorsal p-IPC in approximately half of the samples $(n=22 /$ 41). In the remaining samples, residual NRT-Wg expression was found either in the s-IPC $(n=9 / 41)$, the Nb clone $(n=8 / 41)$, or both $(n=2 / 41)$. The s-IPC and its progeny developed normally, because Hth was expressed and two lobula neuron clusters were formed (Supplementary Fig. $2 \mathrm{~g}-\mathrm{j}$ ). Wg protein and reporter gene expression were detected in the GPC areas before the s-IPC in late 2nd instar larvae (Fig. 2c, d). Furthermore, $w g$ expression in the sIPC of $w g\{K O ; N R T-w g\}$ mid 3rd instar larvae was absent when $w g$ normally is detected (Fig. 2e, f). Thus, GPC-derived Wg could induce $\mathrm{Wg}$ expression in the s-IPC and $\mathrm{Nb}$ clone.

Failure to induce NRT-Wg expression could conceivably not be due to lack of $\mathrm{Wg}$ release but instead to sub-optimal signaling activity of the NRT-Wg protein. We therefore asked whether boosting NRT-Wg expression could overcome observed defects. This was tested by combining a UAS-NRT-wg transgene, known to be highly active ${ }^{33}$ with the knock-in null allele $w g\{K O ; G a l 4\}$, serving also as a driver that restricts activation to endogenous expression domains ${ }^{30}$. UAS-FLP and $w g\left\{K O ; F R T w g^{+}\right.$FRT NRT$w g\}^{30}$ ensured that the endogenous locus only produced NRT-Wg following the induction of recombination events. A tub-Gal80 ts transgene controlled the timing of expression (for full genotypes, see Supplementary Table 1). Thus, at a restrictive temperature, Wg expression is normal and no NRT-Wg is produced. After a shift to a permissive temperature, UAS-NRT-wg is activated and the $w g$ allele switches to expressing NRT-Wg. We found that following a temperature shift at the mid 3rd instar larval stage, NRT-Wg was expressed in the GPC areas and the s-IPC (Fig. 2g; $n=16 / 16$ ). However, following an early temperature shift at the 1st instar larval stage, no expression could be detected in the s-IPC in the majority of samples $(76 \% ; n=26 / 34)$ (Fig. $2 \mathrm{~h})$. We suggest that this partial phenotype is due to imperfect allele switching, i.e., residual wild-type $\mathrm{Wg}$ is produced by the GPC areas, and that $\mathrm{Wg}$ release from the GPC areas is required between the 1st and mid 3rd instar larval stage for inducing Wg expression in the s-IPC. Thus, impaired $\mathrm{Wg}$ release and not reduced signaling activity in $w g\{K O ; N R T-w g\}$ flies accounts for the loss of NRT-Wg expression in secondary domains.

Next, we performed converse allele switching experiments using $w g\left\{K O ; F R T\right.$ NRT-wg FRT $\left.w g^{+}\right\}$to express wild-type Wg in a $w g\{K O ; N R T-w g\}$ background ${ }^{30}$. We used R46E01-Gal4 (Supplementary Fig. $2 \mathrm{k}, \mathrm{l}$ ) and UAS-FLP to induce recombination specifically in the GPC areas. Expression of wild-type $w g$ in the GPC areas rescued the s-IPC-specific loss of NRT-Wg (Fig. 2i, j; $n=16 / 23$ ), demonstrating that $\mathrm{Wg}$ release specifically from the GPC areas is essential.

Consistent with $\mathrm{Wg}$ signaling-dependent induction of $w g$, the target genes frizzled $3(f z 3)$ and notum were expressed in the sIPC (Fig. 2k, 1). Moreover, Wg expression in the s-IPC was abolished following the simultaneous knockdown of the $\mathrm{Wg}$ receptors frizzled $(f z)$ and frizzled $2(f z 2)$ (Fig. $2 \mathrm{~m}, \mathrm{n}$ ) in the IPC by combining the fas3-Gal4 driver $^{23}$ (cf. Supplementary Fig. 7a) with UAS-RNA interference (RNAi) transgenes. Hence, Wg released from the GPC areas is required to induce $w g$ in the s-IPC and $\mathrm{Nb}$ clone (Fig. 2o).

$w g$ is required to induce $d p p$ in adjacent p-IPC subdomains. How can $w g$ in these secondary domains control T4/T5 neurogenesis in the p-IPC/d-IPC? The Drosophila Bmp family member Decapentaplegic (Dpp) is a known target of $w g$ in the OPC and is expressed adjacent to the GPC areas in dorsal and ventral OPC subdomains $^{26}$ (Fig. 3a). Similarly in the IPC, $w g$ expression in the $s$-IPC and Nb clone was detected adjacent to $d p p$-positive ventral and dorsal p-IPC subdomains (Fig. 3b-f). $d p p$ reporter gene expression persisted in the two progenitor streams arising from these subdomains ${ }^{23}$ (Fig. 3c, d). Consistent with stepwise inductive events, $d p p$ in the p-IPC did not precede $w g$ expression in the s-IPC in late 2nd instar larvae (Fig. 3e). Furthermore, $f z 3$ and notum (Fig. 2k, l) were expressed similarly to $d p p$ in p-IPC subdomains and progenitor streams. In $w g\{K O ; N R T-w g\}$ flies, $d p p$-lacZ in the p-IPC was either absent (Fig. $3 g$, h; $n=16 / 32$ ) or showed only residual labeling in one progenitor stream (Supplementary Fig. 3a; $n=16 / 32$ ), in line with the penetrance and expressivity of phenotypes observed in adults. By contrast, OPC expression was unaffected, suggesting that releasable $\mathrm{Wg}$ is not required in this region (Fig. $3 \mathrm{~g}, \mathrm{~h}$ ). Constitutively active $\mathrm{Wg}$ signaling induced by IPC-specific expression of Armadillo ${ }^{\mathrm{S} 10}$ resulted in ectopic $d p p$-lacZ labeling (Fig. 3i; Supplementary Fig. $3 \mathrm{~b})$, confirming that $d p p$ is a Wg target in the p-IPC. IPCspecific $w g$ knockdown abolished $d p p$-lacZ labeling, corroborating that $w g$ is required in the s-IPC and Nb clone, and not the GPC areas for induction (Fig. 3j). Since Dpp signaling mediates EMT of migratory progenitors in the Dpp-expression domains ${ }^{23}$, cell

Fig. $1 \mathrm{Wg}$ release is essential for the formation of lobula plate layers 3/4. a Schematic of the adult Drosophila visual system. Neurons in the lamina (L1/L2) and medulla (e.g., Mi1,4,9, Tm1-4,9) relay ON/OFF motion cues to T4 and T5 neuron dendrites in medulla layer (Me) 10 and lobula (Lo) layer 1. T4/T5 axons innervate lobula plate (Lop) layers 1-4. C\&T neurons include C2/C3 and T2/T3 subtypes. b Schematic of the 3rd instar larval optic lobe. The OPC generates lamina (In) and medulla (mn) neurons. p-IPC NE cells give rise to migratory progenitors that mature into d-IPC Nbs. These produce C\&T and T4/T5 neurons. GMC ganglion mother cells, LPC lamina precursor cells. c Structure of wild-type wg and engineered wg loci (wg $\{K O ; N R T$-wg\}). Open triangles indicate loxP sites. d R9B10-Gal4 UAS-cd8GFP (green) labels T4/T5 neurons. Connectin (red) marks Lop layers 3/4. d-g Neuropils were stained with nc82 (red) and aPKC (blue). Compared to controls (e), in wg $\{K O ; N R T$-wg $\}$ flies, one (f) or two (g) lobula plate layers were absent. $\mathbf{h}$ The decrease of layers correlates with T4/T5 neuron numbers. The scatter plot with bars shows data points and means with $\pm 95 \%$ confidence interval error bars $(n=15$; three optical sections from five samples per genotype). Unpaired, two-tailed Student's t-test not assuming equal variance: $P=4.72 \times 10^{-11}$ and $P=3.23 \times$ $10^{-17}$. ${ }^{\star \star \star \star} P<0.0001$. Unlike in controls (i), Connectin was found in one (j) or none (k) of the Lop layers in $w g\{K O ; N R T$-wg $\}$ flies. Similar to nc82 (f), Connectin labeling showed gaps in the third lobula plate layer (j), potentially consisting of both layer 3 and 4 neurons. I-n In wild-type 3rd instar larvae (3L), the GPC areas (arrowheads), surface (s-)IPC (dashed line, double arrowheads), and a Nb clone (arrow) adjacent to the dorsal p-IPC subdomain (dashed line) express Wg (green). o Schematics of larval Wg expression (blue) in 3D and a horizontal section. Arrow indicates Nb lineage. For genotypes and sample numbers, see Supplementary Table 1. Scale bars, $50 \mu \mathrm{m}$ 
streams from these areas were affected in $w g\{K O ; N R T-w g\}$ flies and following IPC-specific knockdown of $w g$ and the Dpp type I receptor thickveins (tkv) (Fig. $3 \mathrm{~h}, \mathrm{j}, \mathrm{k}$ ). Although the overall d-IPC morphology was altered (Supplementary Fig. 3c-f), progenitors and $\mathrm{Nbs}$ generated from the remaining $\mathrm{p}$-IPC showed wild-type marker expression (Supplementary Fig. $3 g-n$ ). In adults, similar to $w g\{K O ; N R T-w g\}$ flies, lobula plate layers $3 / 4$ were absent following IPC-specific knockdown of $f z, f z 2$, or tkv (Fig. 31-n).
Hence, $w g$ from the s-IPC and Nb clone regulates $d p p$ expression in adjacent p-IPC subdomains and EMT of progenitors that mature into Nbs producing T4/T5 neurons for layers 3/4 (Fig. 3o).

Layer 3/4 T4/T5 neurons arise from Dpp-positive subdomains. To provide evidence that T4/T5 neurons innervating layers 3/ 4 specifically originated from the Dpp-positive subdomains in the
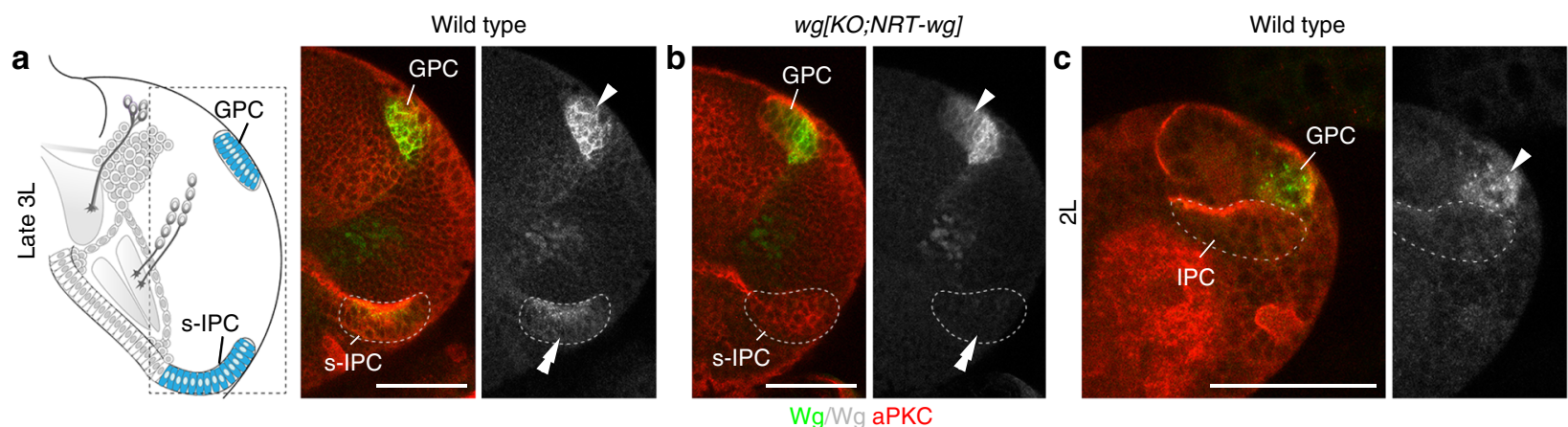

$w g[K O ; G a l 4]>c d 8 G F P$

$w g[K O ; G a l 4]>c d 8 G F P, w g[K O ; N R T-w g]$

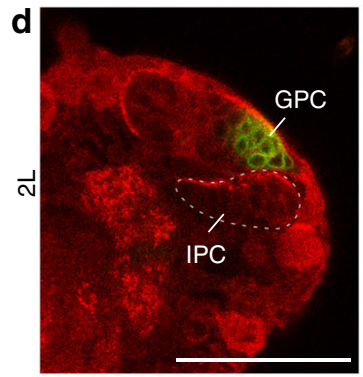

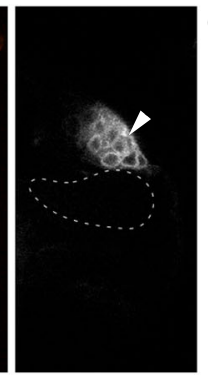

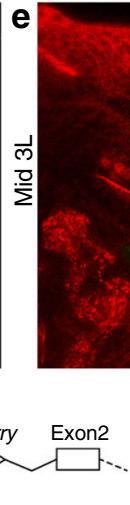

tub-Gal80 ts, wg[KO;Gal4] > UAS-NRT-wg, UAS-FLP
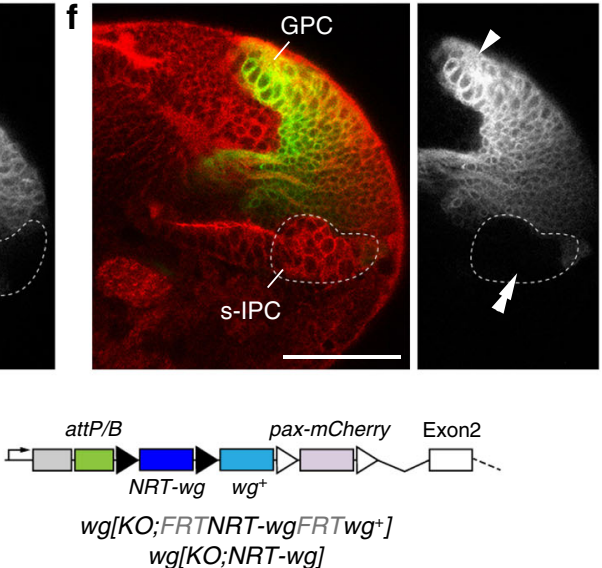

$w g[K O ; N R T-w g]$
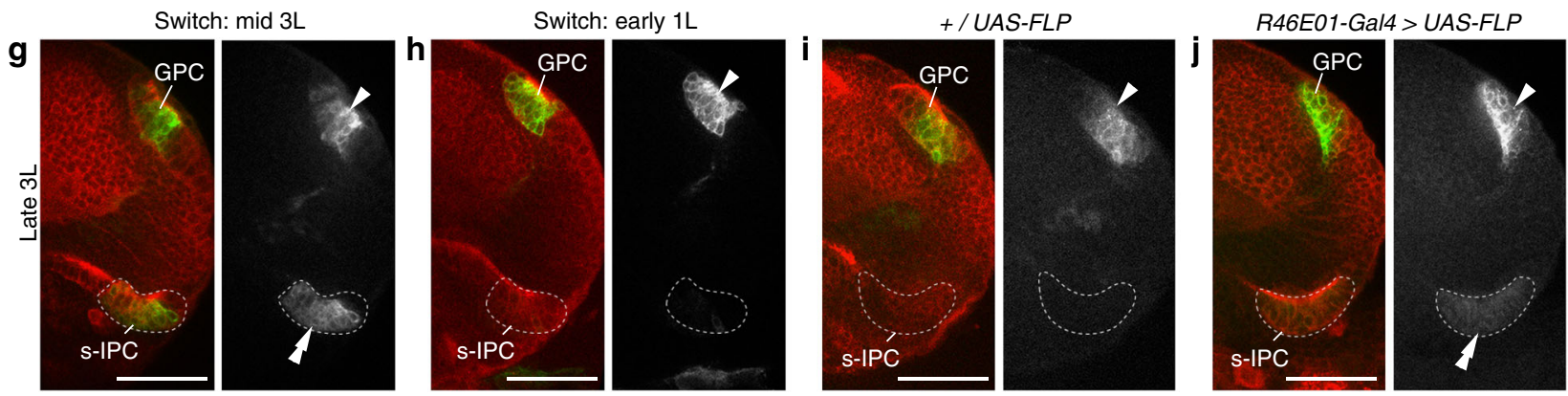

Wg/Wg aPKC

$f z 3-G F P$

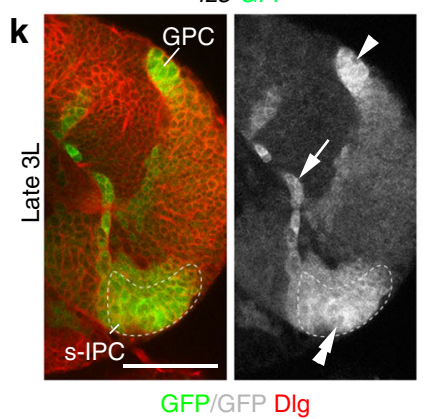

notum-lacZ

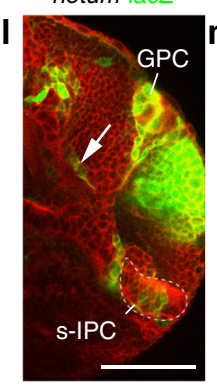

$\beta \mathrm{Gal}$ aPKC

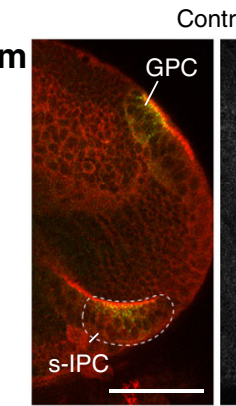

Control

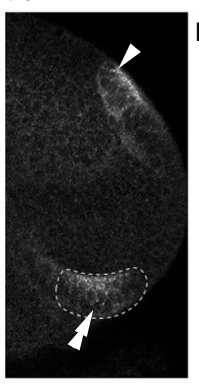

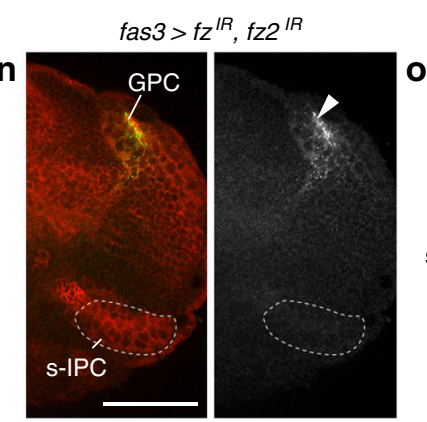

o

GPC

wg

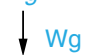

S-IPC/Nb clone

wg 
p-IPC, we next conducted lineage-tracing experiments. Because $d p p$-Gal4 is expressed in the entire optic lobe, we searched for a driver with activity restricted to the IPC Dpp-expression domains. Expression of R45H05-Gal4 (Fig. 4a) is controlled by an enhancer fragment of dorsocross 1 , which encodes a T-box transcription factor and known Dpp target gene ${ }^{34}$. Reporter gene expression was detected in dorsal and ventral p-IPC subdomains, cell streams, Nbs, and postmitotic progeny in larval optic lobes and persisted throughout pupal development into adulthood, where it specifically labeled T4/T5 neurons innervating the layers $3 / 4$ but not $1 / 2$ (Fig. 4 b; Supplementary Fig. $4 a$, b). As the expression was weak, we combined the FLPout approach with tub-Gal80 ${ }^{t s}$ to permanently label $\mathrm{R} 45 \mathrm{H} 05-\mathrm{Gal} 4$ expressing progeny, and again solely detected $\mathrm{T} 4 / \mathrm{T} 5$ neurons projecting into layers 3/4 (Fig. 4c). Finally, we used the Flybow approach to label lineages in different colors. All clones contained T4/T5 neurons innervating either layers $1 / 2$ or $3 / 4$ but not both (Fig. $4 \mathrm{~d} ; n=43$ clones in 26 optic lobes), consistent with distinct origins of these neuron subtypes.

In lineage-tracing experiments using R45H05-Gal4, clones included $\mathrm{C} 2$, the T2-variant T2a, and/or T3 neurons, as judged by their characteristic cell body positions and terminals in the lamina and lobula, respectively (Fig. 4e). While C3, T2, and T3 neurons were not affected, C2 neurons were fully or partially absent in $w g\{K O ; N R T-w g\}$ flies with two or three remaining lobula plate layers, respectively (Fig. 4f, g; Supplementary Fig. $4 c-j)$. Due to the lack of specific markers, we could not conduct tests for T2a neurons. Thus, T4/T5 neurons innervating layers $3 / 4, \mathrm{C} 2$, and possibly $\mathrm{T} 2 \mathrm{a}$ neurons are specifically derived from the dorsal and ventral Dpp-expression domains, while the p-IPC core generates T4/T5 for layers $1 / 2$, as well as C3, T2, and T3 neurons (Fig. 4h).

dac and ato mediate the transition to T4/T5 neuron formation. We next asked when and how layer-specific T4/T5 neurons become distinct during development. Dac and Ato are expressed in the second $\mathrm{d}$-IPC $\mathrm{Nb}$ competence window ${ }^{23}$. While Dac is initially expressed in all T4/T5 neurons in 3rd instar larvae, albeit with varying expression levels, Dac was only found in $~ 50 \%$ of adult T4/T5 neurons (Fig. 5a-c; see below). The enhancer trap dac ${ }^{7 d 23}$-Gal4 line faithfully reported Dac expression in T4/T5 neurons throughout development and remained active in neurons innervating layers $1 / 2$ (Fig. 5d, e; Supplementary Fig. 5a). Thus, Dac is downregulated in layer 3/4 innervating T4/T5 neurons and maintained in neurons projecting to layers $1 / 2$ (Fig. $5 f$ ).

To examine the function of $d a c$, we used mosaic analysis with a repressible cell marker (MARCM) to generate $d a c^{1}$ mutant T4/T5 neurons labeled with the dac enhancer Gal4 line R9B10-Gal4 (Supplementary Fig. 5b, c). Some adult dac mutant neurons showed T2/T3-like morphologies with neurites extending into the medulla layer M9 and higher, and synaptic terminals in lobula layers Lo2 and Lo3 (Fig. 5f-j). This suggested that mutant neurons adopted features of neurons born in the first d-IPC Nb competence window. To assess potential redundancy, we performed $d a c$ and ato knockdown experiments using validated $U A S-R N A i$ transgenes (Supplementary Fig. 5d-g). In samples with IPC-specific single dac or ato knockdown, frequently only one Connectin-positive lobula plate layer was discernible (Supplementary Fig. 5h-j). However, simultaneous dac and ato knockdown caused the absence of neurons with T4/T5 morphologies and in consequence an undersized lobula plate neuropil. Fas 3 in T4/T5 dendrites within medulla layer Me10 and lobula layer Lo1 and Connectin in lobula plate layers were severely reduced. Consistent with T2/T3-like morphologies, remaining neurons innervated the medulla and lobula (Fig. 5k-n; Supplementary Fig. 5k).

Therefore, dac and ato are required together for the switch from T2/T3 to T4/T5 neuron formation. Dac is maintained in layer $1 / 2$ innervating T4/T5 neurons, but downregulated in layer $3 / 4$ innervating T4/T5 neurons, suggesting that layer $1 / 2$ identity could represent the default fate.

Notch controls the choice between T4 and T5 neuron identity. In the OPC, Notch signaling in asymmetric GMC divisions contributes to generating neuronal diversity ${ }^{35}$, involving differential apoptosis in region-specific lineages ${ }^{36}$. We therefore assessed whether this pathway could mediate the distinction of layer 1/2 and 3/4 innervating T4/T5 neurons downstream of Dpp. We did not detect apoptotic cells in the 3rd instar larval lobula plate (Supplementary Fig. 6a). However, expression of an activated, ligand-independent form of Notch $\left(\mathrm{N}^{\mathrm{intra}}\right)$ in d-IPC Nbs in the second competence window and their progeny using the late R9B10-Gal4 driver affected T4 neuron formation, because neurites were missing in the medulla of 3rd instar larvae (Fig. 6a, b). Adults exhibited a milder phenotype, in which mostly T4 neurons connecting to the anterior proximal medulla were affected (Supplementary Fig. 6b-h). Conversely, IPC-specific knockdown of the transcriptional regulator Suppressor of Hairless $(\mathrm{Su}(\mathrm{H}))$ using R17B05-Gal4 caused the absence of T5 neurons in adults, whereas T4 neurons were present (Fig. 6c, d; Supplementary Fig. 6f). In these flies, lobula plate layers 3 and 4 could not be discriminated (Supplementary Fig. 6i). The lobula plate and lobula neuropils were severely disorganized, likely because of an early requirement of Notch in p-IPC NE cells during the 3rd instar larval stage ${ }^{27}$ (Supplementary Fig. 6j, k). Hence, Notch controls the choice between T4 and T5 neuron fate (Fig. 6e), whereas the distinction between layer $1 / 2$ Dac-positive and layer $3 / 4$ Dac-negative T4/T5 neurons is mediated by a Notchindependent mechanism.

Dpp-dependent specification of layer 3/4 T4/T5 neurons by $\boldsymbol{o m} \boldsymbol{b}$. While $d p p$ reporter gene activity extended from the p-IPC

Fig. 2 The GPC areas release Wg to induce $w g$ in the s-IPC. a, b Schematic in a highlights the region of interest shown in subsequent panels. Unlike in controls (a), Wg immunolabeling (green) was absent in the s-IPC (dashed line, double arrowhead) in many wg $\{K O ; N R T$-wg $\}$ flies (b). The GPC areas (arrowhead) were not affected. c, d In 2nd instar larvae (2L), Wg protein (c, green) and wg \{KO;Gal4\} UAS-cd8GFP (d, green) were detected in GPC areas (arrowhead), but not in the adjacent IPC (dashed line). e, f s-IPC-specific wg $\{K O ; G a l 4\}$ UAS-cd8GFP expression (green, double arrowheads) in mid 3rd instar larvae (e) was absent in wg $\{K O ; N R T$-wg $\}$ flies (f). Arrowheads indicate expression in GPC area. $\mathbf{g}, \mathbf{h}$ In controls, wg $\{K O ; G a l 4\}$ UAS-FLP mediated $w g\left\{K O ; F R T\right.$ wg ${ }^{+}$FRT NRT-wg $\}$allele switching and simultaneous UAS-NRT-wg overexpression were induced at the mid 3rd instar larval stage (g, wg ${ }^{+}$ background). Allele switching at the 1st instar larval stage (h, NRT-wg background) did not rescue s-IPC-specific Wg loss (green). i, $\mathbf{j}$ Unlike in controls (i), R46E01-Gal4 UAS-FLP-mediated GPC areas-specific wg $\left\{K O ; F R T N R T\right.$-wg FRT $\left.w g^{+}\right\}$allele switching (j) rescued s-IPC-specific NRT-Wg loss (green). Filled and open triangles in transgene schematics represent $F R T$ and loxP sites, respectively $(\mathbf{g}-\mathbf{j})$. $\mathbf{k}, \mathbf{I}$ The Wg target gene reporter lines $f z 3 G 00357$-GFP (k, green) and notum ${ }^{W R E}$-lacZ (I, green) show expression in the GPC areas, the s-IPC, and in migratory progenitors (arrow) originating from the adjacent $\mathrm{p}-\mathrm{IPC}$. $\mathbf{m}, \mathbf{n}$ Unlike in controls (m), fas $3^{\mathrm{NP1233}}$-Gal4-mediated IPC-specific $f z$ and $f z 2$ knockdown (n) caused loss of Wg (green) in the s-IPC. o Summary of wg function in the GPC areas. For genotypes and sample numbers, see Supplementary Table 1. Scale bars, $50 \mu \mathrm{m}$ 

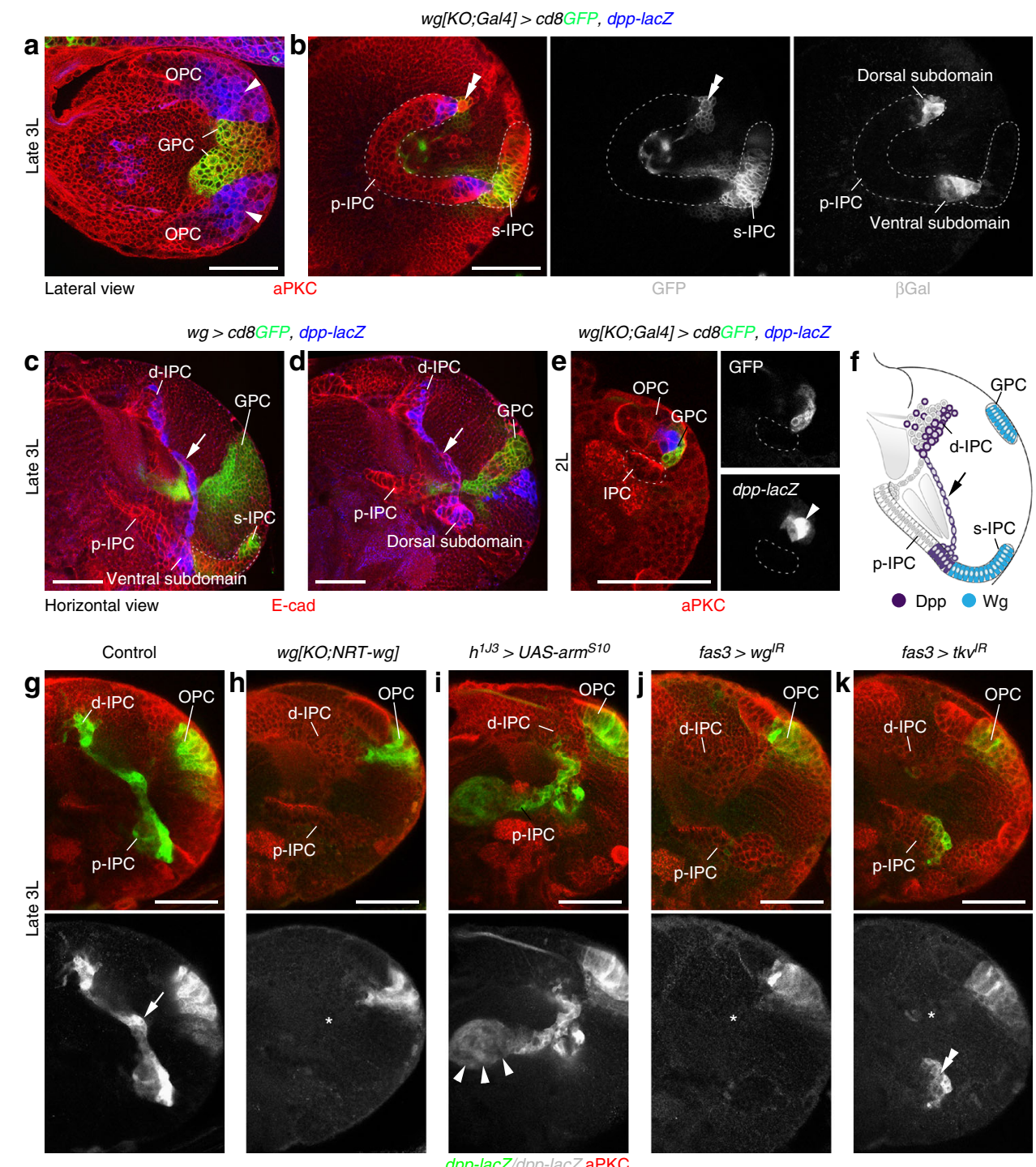

$w g[K O ; G a / 4]>c d 8 G F P, d p p-l a c Z$
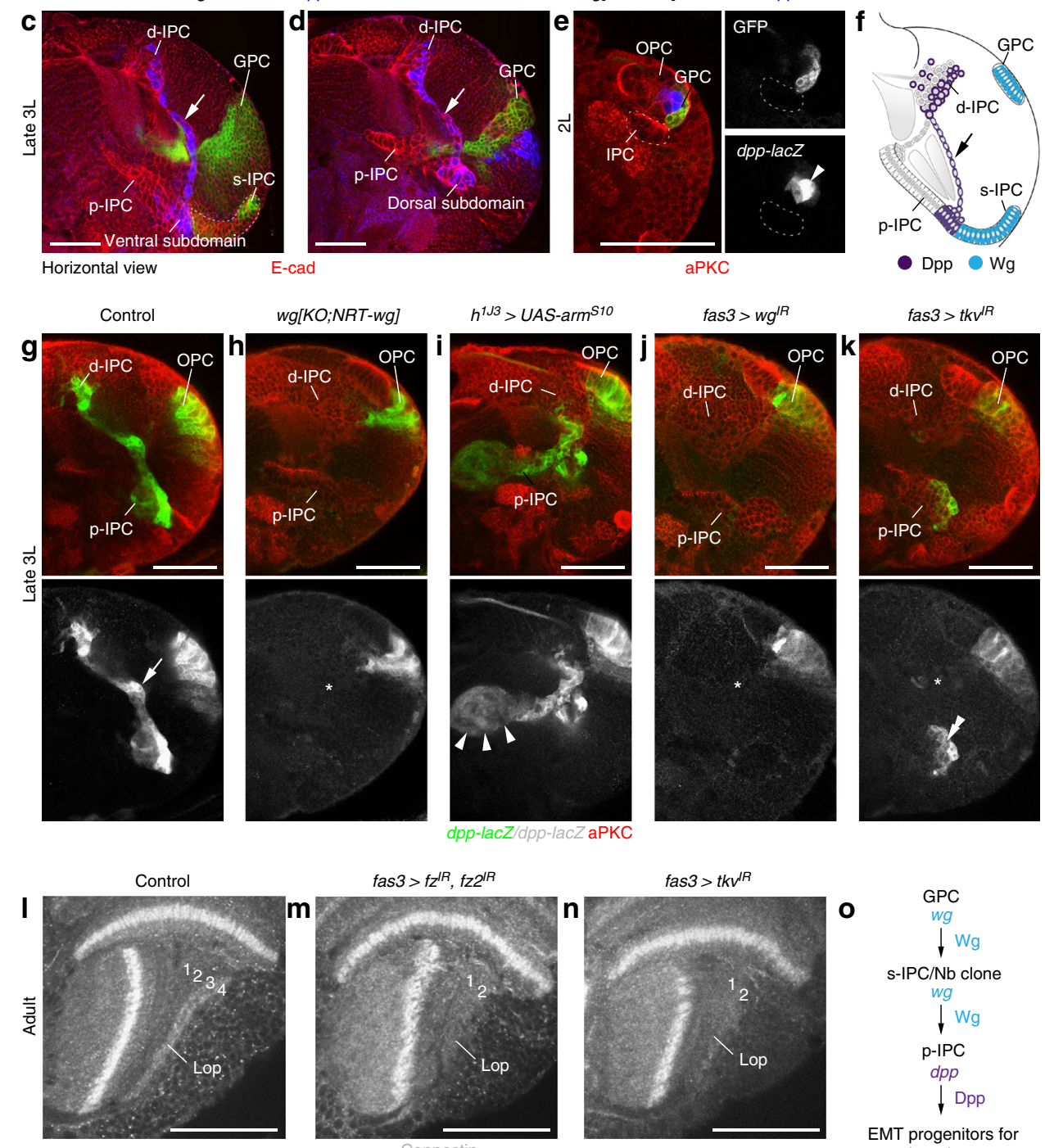

EMT progenitors for layer 3/4 T4/T5 neurons

Fig. 3 s-IPC-derived Wg is required for Dpp-dependent EMT in the p-IPC. a, b In 3rd instar larvae (3L), dpp-lacZ (blue) was detected in subdomains adjacent to $w g\{K O ; G a l 4\}$ UAS-cd8GFP (green) regions in the dorsal and ventral OPC (a, arrowheads) and p-IPC (b). Double arrowhead indicates GFPpositive $\mathrm{Nb}$ clone adjacent to the dorsal p-IPC. c, $\mathbf{d}$ dpp-lacZ (blue) was maintained in progenitor streams (arrows) from the ventral and dorsal p-IPC subdomains. e In 2nd instar larvae (2L), dpp-lacZ (blue) was present in the OPC (arrowhead) adjacent to wg \{KO;Gal4\} UAS-cd8GFP-positive GPC areas (green), but was absent in the IPC (dashed line). f Schematic illustrating Wg and Dpp expression domains. Arrow indicates progenitor stream originating from the ventral p-IPC subdomain. $\mathbf{g}$-k Unlike in controls ( $\mathbf{g}$, arrow), dpp-lacZ (green) was absent from the IPC in wg $\{K O ; N R T$-wg $\}$ flies (h, asterisk). The OPC was not affected. dpp-lacZ was ectopically induced in the IPC (arrowheads) by $h^{1 / 3}$-Gal4-mediated expression of UAS-arm ${ }^{S 10}$ (i). dpp-lacZ was absent following fas3 ${ }^{N P 1233}$-Gal4-mediated IPC-specific wg knockdown. In the Dpp-expression domain, this caused EMT defects and loss of progenitor streams ( $\mathbf{j}$, asterisks). Similar defects were caused by IPC-specific tkv knockdown. dpp-lacZ remains expressed in the p-IPC (k, double arrowheads).

I-n Compared to controls (I), fas $3^{N P 1233}$-Gal4-mediated IPC-specific knockdown of $f z$ and $f z 2$ ( $\mathbf{m}$ ) and tkv (n) caused the loss of lobula plate (Lop) layers $3 / 4$ labeled with Connectin in adults. o Summary of $w g$ and $d p p$ function in the GPC areas, the s-IPC/Nb clone, and p-IPC. For genotypes and sample numbers, see Supplementary Table 1. Scale bars, $50 \mu \mathrm{m}$ 


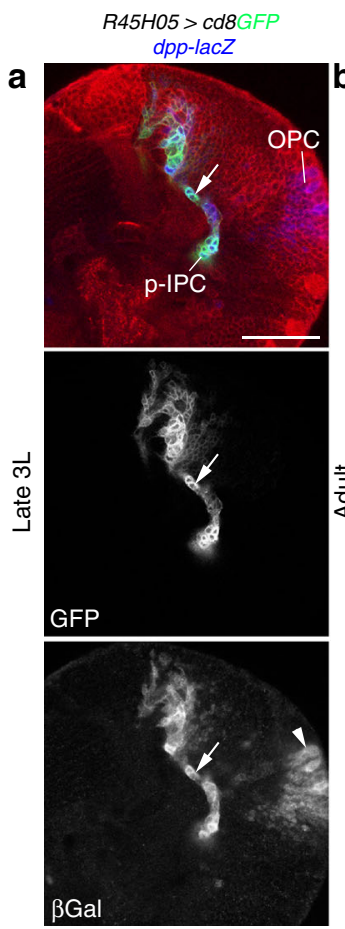

GFP aPKC $\beta$ Gal

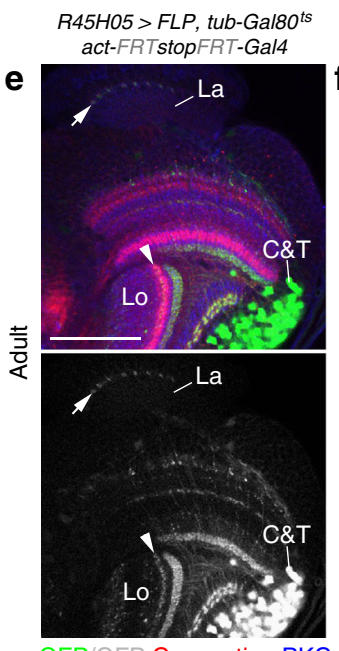

GFP/GFP Connectin aPKC
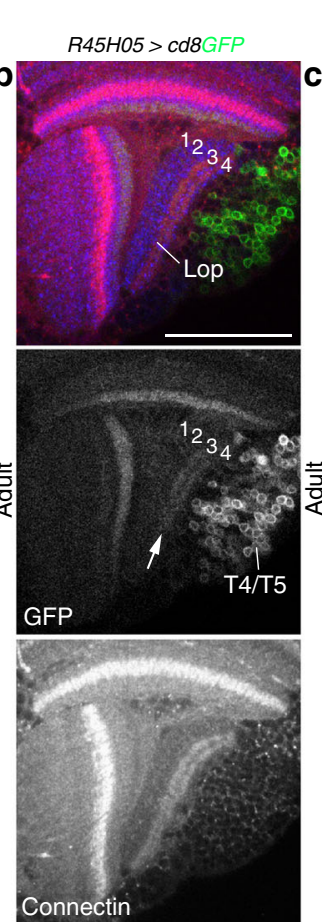

GFP Connectin aPKC

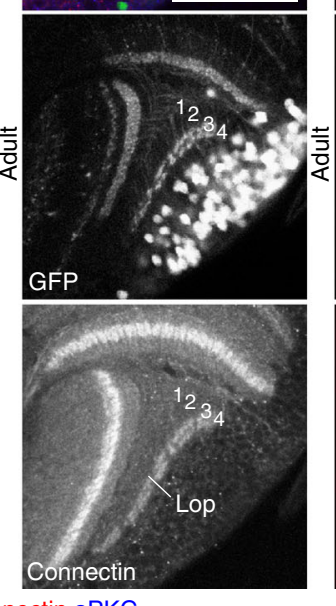

R45H05 > FLP, tub-Gal80
act-FRTstopFRT-Gal4
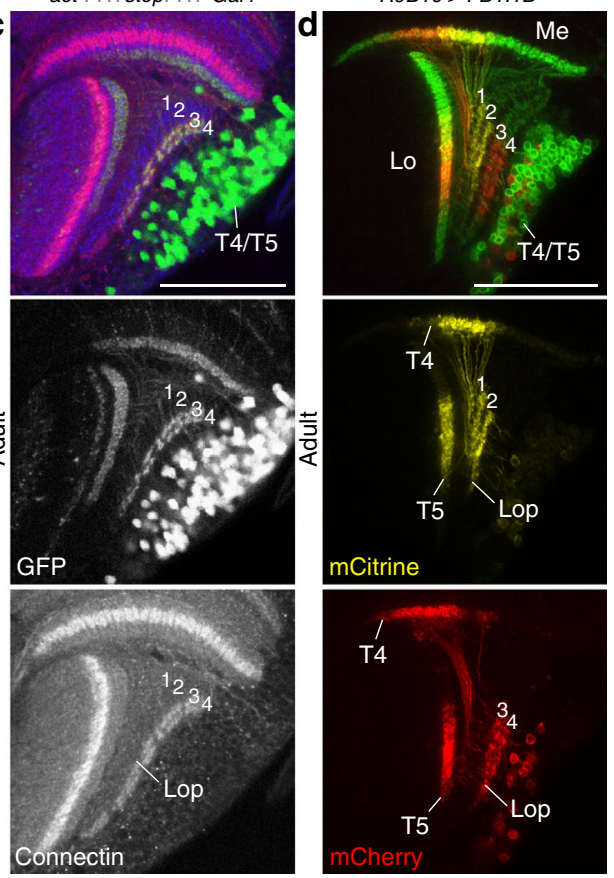

GFP mCitrine mCherry

h
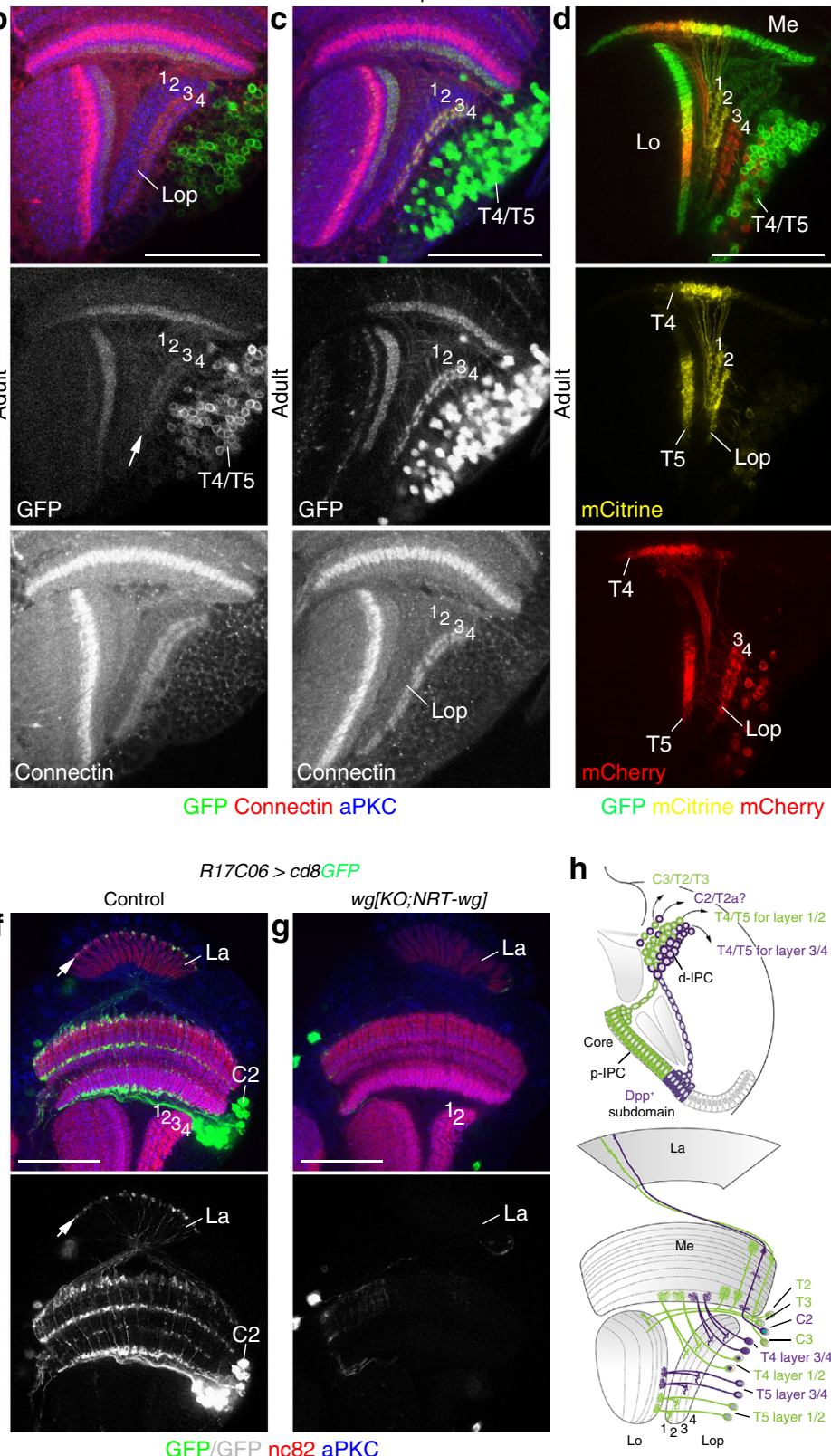

$\mathrm{Dpp}^{+}$
subdomain

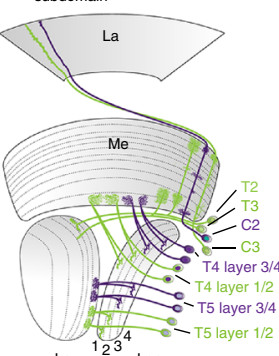

Fig. 4 The Dpp-expression domain generates C2 and T4/T5 neurons for layers 3/4. a R45H05-Gal4 UAS-cd8GFP (green) colocalizes with the dpp-lacZ (blue) expression domain in the IPC (arrow) but not the OPC (arrowhead) in 3rd instar larvae (3L). b R45H05-Gal4 UAS-cd8GFP (green) was weakly maintained in T4/T5 neurons innervating Connectin-positive lobula plate (Lop) layers 3/4 (red, arrow) in adults. c Permanent GFP-labeling (green) of the R45H05-Gal4 expression domain using act $>y^{+}>$Gal4 UAS-GFP, UAS-FLP, and tub-Gal80 ts was specific to T4/T5 neurons innervating lobula plate layers $3 / 4$. d R9B10-Gal4 in conjunction with Flybow transgenes labeled T4/T5 neuron clones either innervating lobula plate (Lop) layers 1/2 (mCitrine, yellow) or $3 / 4$ (mCherry, red). e Permanent GFP labeling (green) with R45H05-Gal4 included C2, as well as T2a and/or T3 neurons with characteristic axon terminals in the lamina (La, arrow) and lobula (Lo, arrowhead). f, $\mathbf{g}$ Unlike in controls (f), C2 neuron-specific R17C06-Gal4 UAS-cd8GFP expression (green) with axon terminals (arrow) in the lamina (La) was absent in $w g\{K O ; N R T$-wg $\}$ flies that had two remaining lobula plate layers (g). $\mathbf{h}$ Schematics illustrating the neuron subtypes derived from the Dpp-expression domain and the core p-IPC in 3rd instar larvae and adults. For genotypes and sample numbers, see Supplementary Table 1. Scale bars, $50 \mu \mathrm{m}$

to the d-IPC, phospho-Mad (pMad) labeling was restricted to $\mathrm{p}$ IPC NE cells (Fig. 7a). Consistently, knockdown experiments using Gal4 lines with progressively restricted activities (Supplementary Fig. $7 \mathrm{a}-\mathrm{c}$ ) revealed a requirement of $t k v$ for layer $3 / 4$ neuron formation in p-IPC NE cells (cf. Fig. 3n), but not in d-IPC Nbs and T4/T5 neurons (Supplementary Fig. 7d-f). Therefore, an additional mechanism must relay Dpp signaling activity in p-IPC
NE cells to distant postmitotic T4/T5 neurons. The T-box transcription factor Optomotor blind $(\mathrm{Omb})$ is a Dpp target in the $\mathrm{p}-\mathrm{IPC}^{23}$. In 3rd instar larval brains, Omb expression is maintained in progenitors, Nbs, and T4/T5 neurons derived from the Dpp-positive p-IPC subdomains (Fig. 7b, c). Expression persisted in adult T4/T5 neuron subsets (Supplementary Fig. 7g). In $w g\{K O ; N R T-w g\}$ flies, omb expression was severely reduced in 
the p-IPC and progeny, but not in the OPC (Fig. 7d, e). Consistently, Omb was also decreased following IPC-specific tkv knockdown (Fig. 7f, g).

To determine the function of omb, we conducted knockdown experiments using fas3-Gal4 and two validated UAS-RNAi transgenes (Supplementary Fig. $7 \mathrm{~h}-\mathrm{k}$ ). Experimental animals were raised at $18^{\circ} \mathrm{C}$ and shifted to $29^{\circ} \mathrm{C}$ at the early 3 rd instar larval stage, because omb is expressed in the embryonic optic lobe placode and null mutations cause severe disorganization or complete loss of adult optic lobes ${ }^{37}$. Under these conditions, omb knockdown did neither affect $d p p$ expression nor the EMT of progenitors from the p-IPC (Fig. 7h, i). However in adults, lobula plate layers $3 / 4$ were absent (Fig. $7 \mathrm{j}, \mathrm{k}$ ). In contrast to $t k v$, omb knockdown using Gal4 lines with progressively restricted activities revealed that this transcription factor is required in $\mathrm{Nb}$ in the second competence window and postmitotic $\mathrm{T} 4 / \mathrm{T} 5$

$R 9 B 10>c d 8 G F P$
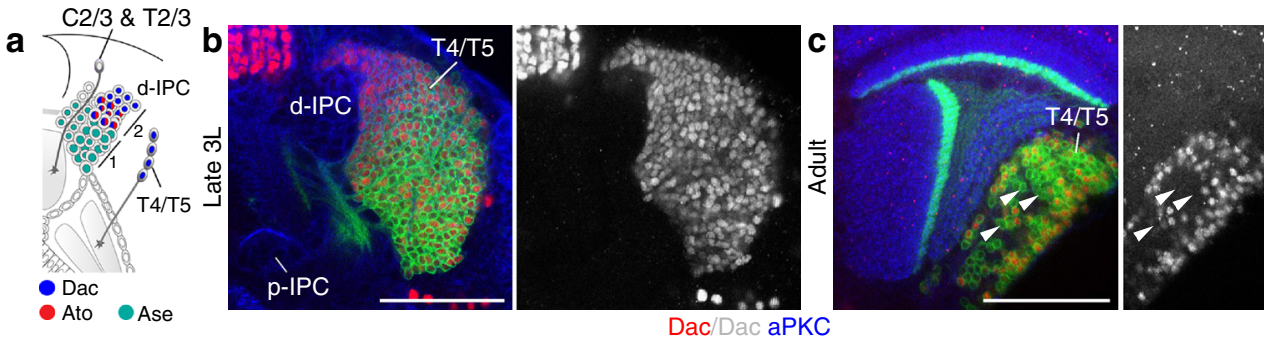

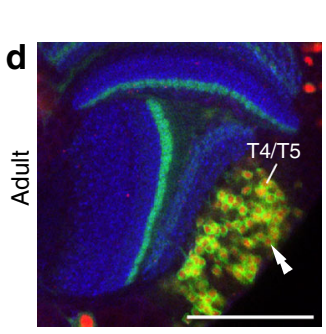

Dac aPKC

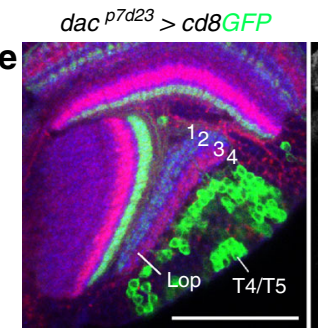

GFP/GFP Connectin aPKC
Dac

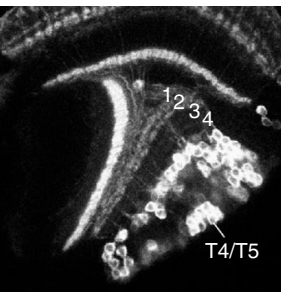

$R 9 B 10>$ Cd8GFP
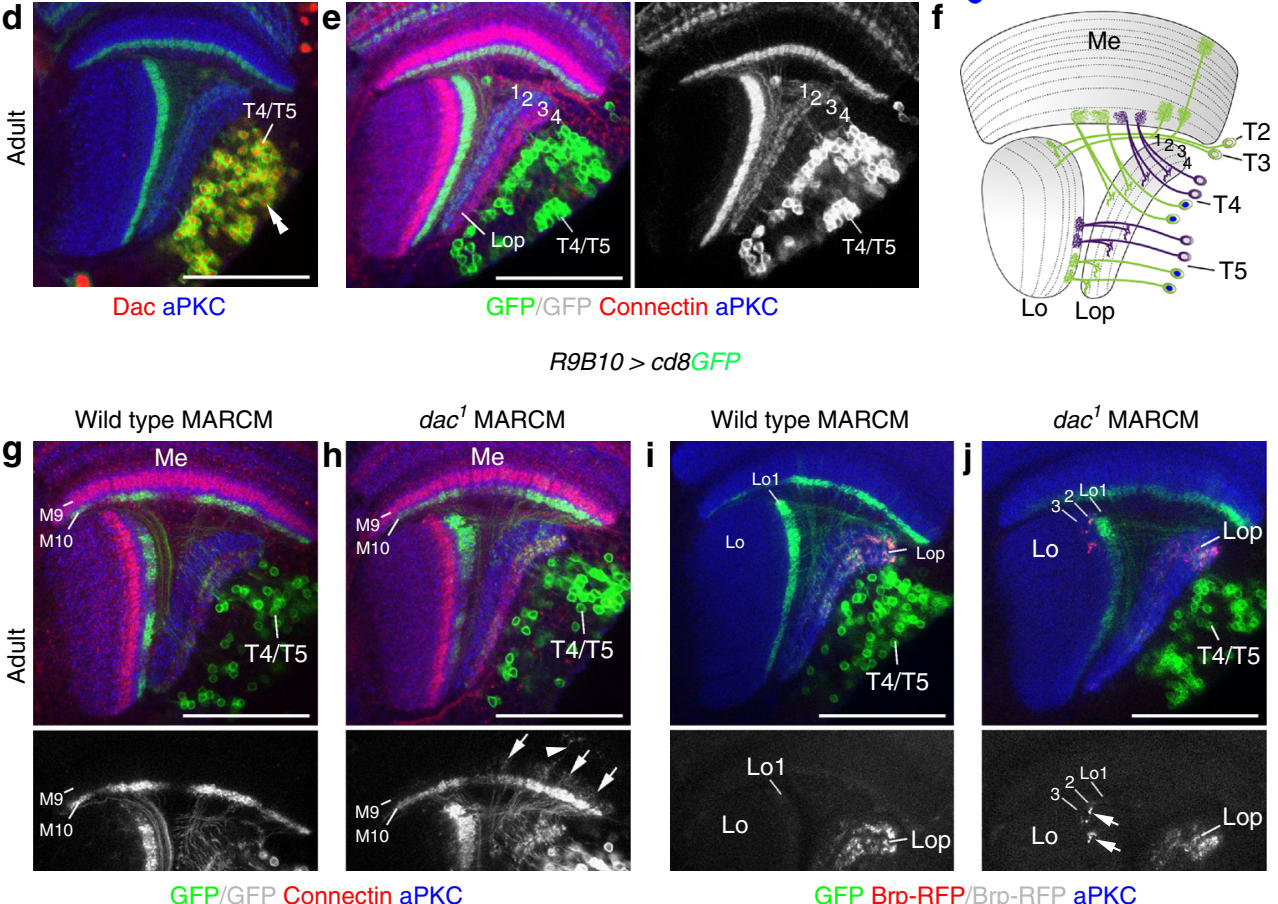

Lo Lop

fas3 $>$ cd8GFP
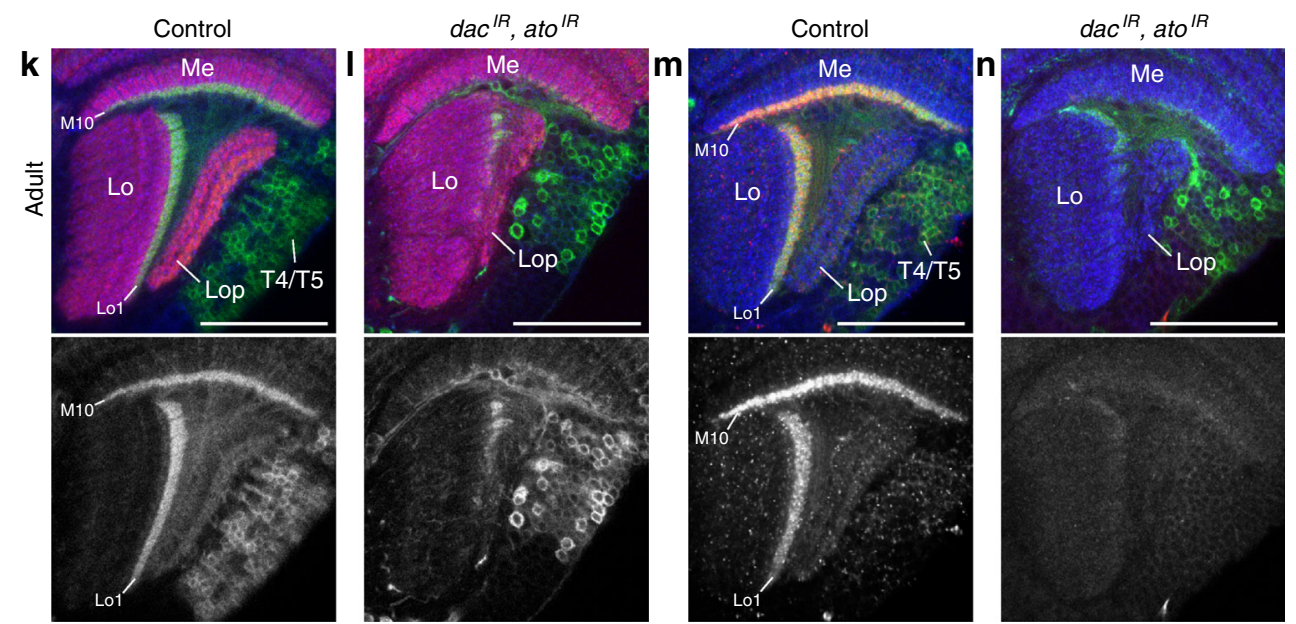

GFP Fas3/Fas3 aPKC 
neurons for layer $3 / 4$ neuron specification (Fig. 7l, m). Thus, omb could relay subdomain-specific Dpp signaling effects in p-IPC NE cells across intermediate cellular states, i.e., migratory progenitors, Nbs, and T4/T5 progeny (Fig. $7 \mathrm{n}$ ).

omb mediates layer 3/4 T4/T5 neuron specification by Dac repression. Finally, we assessed whether Omb could be responsible for downregulating Dac. In 3rd instar larvae, Omb was coexpressed with high levels of Dac in younger and low levels in older T4/T5 neurons (Fig. 8a). From $24 \mathrm{~h}$ after puparium formation (APF) (Fig. 8b) to adulthood (Supplementary Fig. 8a), Omb and Dac showed mutually exclusive expression in T4/T5 neurons. While Dac was maintained in $~ 50 \%$ of adult T4/T5 neurons in controls (Fig. 8c, h), 95\% of T4/T5 neurons expressed Dac and adopted layer $1 / 2$ identity following omb knockdown (Figs. $7 \mathrm{~m}, 8 \mathrm{~d}, \mathrm{~h}$ ). Conversely, when $o m b$ was overexpressed, none of the T4/T5 neurons expressed Dac and all acquired layer 3/4 identity (Fig. 8e, h, j). Under both conditions, many T4/T5 neurons underwent apoptosis in 3rd instar larvae (Supplementary Fig. $8 \mathrm{~b}-\mathrm{d}$ ). Consistently in adults, their numbers were reduced by $33 \%$ and $48 \%$, respectively (Fig. $8 \mathrm{~h}$ ), possibly because excessive T4/T5 neurons either for layers $1 / 2$ or $3 / 4$ compete for limited trophic support. To demonstrate that omb is sufficient, we took advantage of $w g\{K O ; N R T-w g\}$ flies, in which all T4/T5 neurons adopted layer $1 / 2$ identity and Dac was expressed in $97 \%$ of these (Fig. 8f, h; Supplementary Fig. 8e, f). In this background, omb overexpression only mildly affected neuron numbers $(19 \%$ reduction) and Dac was downregulated (Fig. 8g, h). Importantly, concomitant upregulation of Connectin suggested that these differentiated into layer 3/4 innervating T4/T5 neurons (Fig. 8i, k). Thus, omb is required and sufficient for specifying T4/T5 neurons innervating layers $3 / 4$ by downregulating Dac (Fig. 8l).

\section{Discussion}

The spread of $\mathrm{Wg}$ is dispensable for patterning of many tissues ${ }^{30}$. However, our study uncovered a distinct requirement for diffusible $\mathrm{Wg}$ in the nervous system, where it orchestrates the formation of T4/T5 neurons innervating lobula plate layers 3/4. Their generation depends on inductive mechanisms (Fig. $8 \mathrm{~m}$ ) that are relayed in space and time. The spatial relay consists of a multistep-signaling cascade across several NE domains: Wg from the GPC areas induces $w g$ expression in the s-IPC and $\mathrm{Nb}$ lineage adjacent to ventral and dorsal p-IPC subdomains; this secondary Wg source activates $d p p$ expression. Dpp signaling mediates EMT of migratory progenitors from these subdomains. The p-IPC core produces Dac-positive layer 1/2 specific T4/T5 neurons. Dpp signaling in p-IPC NE subdomains triggers a temporal relay across intermediate cellular states by inducing $0 \mathrm{mb}$. Omb in turn suppresses Dac, conferring layer 3/4 identity to postmitotic T4/T5 neurons.

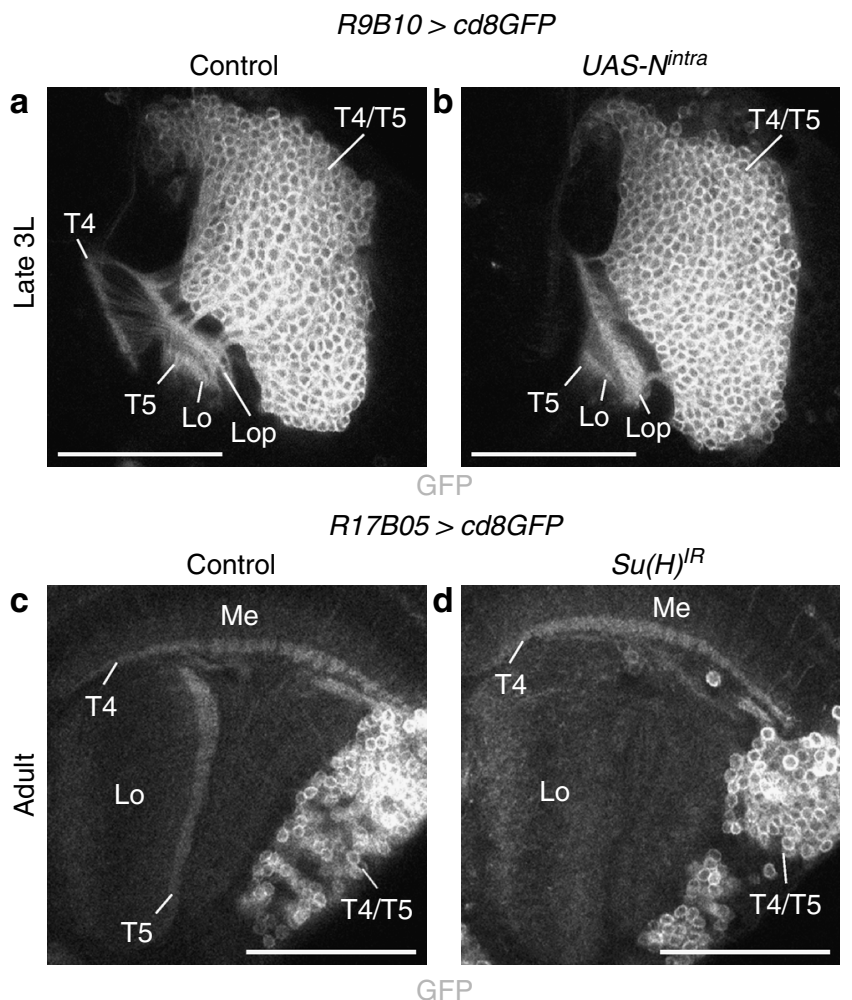

e

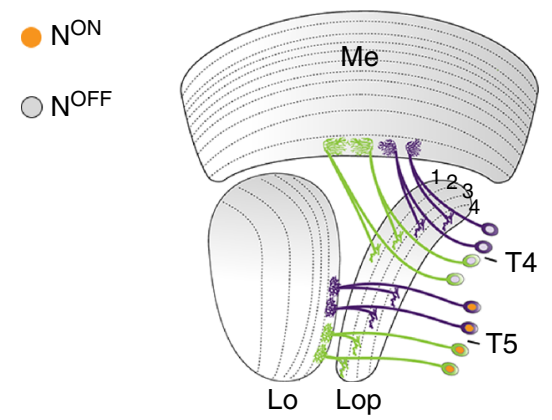

Fig. 6 Notch controls the choice between T4 and T5 neuron fate. a, b Unlike in controls (a), R9B10-Gal4 UAS-cd8GFP labeled T4 neurites were absent in 3rd instar larvae following UAS-N ${ }^{\text {intra }}$ overexpression in $\mathrm{d}$-IPC Nbs and their progeny during the second competence window (b). T5 neurites in the lobula (Lo) were unaffected. c, d Unlike in controls (c), following R17B05Gal4 UAS-cd8GFP-mediated IPC-specific Su(H) knockdown, T5 neurites were absent in the adult lobula (d). T4 neurites were present. e Schematic illustrating the Notch-dependent specification of T5 neurons. For genotypes and sample numbers, see Supplementary Table 1. Scale bars, $50 \mu \mathrm{m}$

Fig. 5 dac and ato are required for T4/T5 neuron formation. a Schematic illustrating the expression of Ase (turquoise), Ato (red), and Dac (blue) in d-IPC Nbs during the first (1) and second (2) competence windows and their progeny. b, c Dac (red) was expressed in all R9B10-Gal4 UAS-cd8GFP (green)

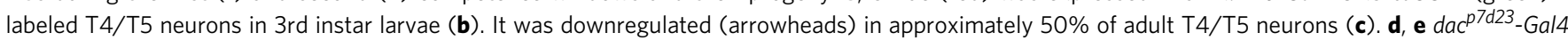
UAS-cd8GFP (green) faithfully reported Dac (red, double arrowhead) expression in adults (d), specifically labeling T4/T5 neurons innervating lobula plate (Lop) layers 1 and 2 (e). f Schematic illustrating Dac expression (blue) in adult T4/T5 neurons. $\mathbf{g}$-j Unlike in controls (g, i), dac ${ }^{1}$ mutant T4/T5 neurons adopted T2/T3 neuron morphologies with R9B10-Gal4 UAS-cd8GFP (green), displaying neurite extensions into medulla (Me) layer M9 (arrows) and more distal layers (arrowhead) (h), and UAS-brp-RFP labeled synaptic terminals (red, arrows) in lobula (Lo) layers 2 and 3 (j). k, I Unlike in controls (k), fas3 ${ }^{N P 1233}$-Gal4 UAS-cd8GFP (green)-mediated IPC-specific simultaneous knockdown of dac and ato generated neurons that failed to form a four-layered lobula plate (Lop) neuropil and dendrites in medulla (Me) layer 10 and lobula (Lo) layer 1 (I). m, n Unlike in controls (m), Fas3-positive (red) T4/T5

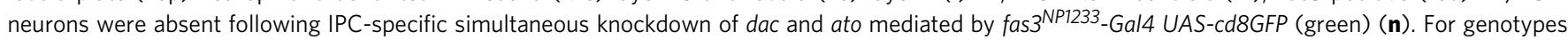
and sample numbers, see Supplementary Table 1. Scale bars, $50 \mu \mathrm{m}$ 


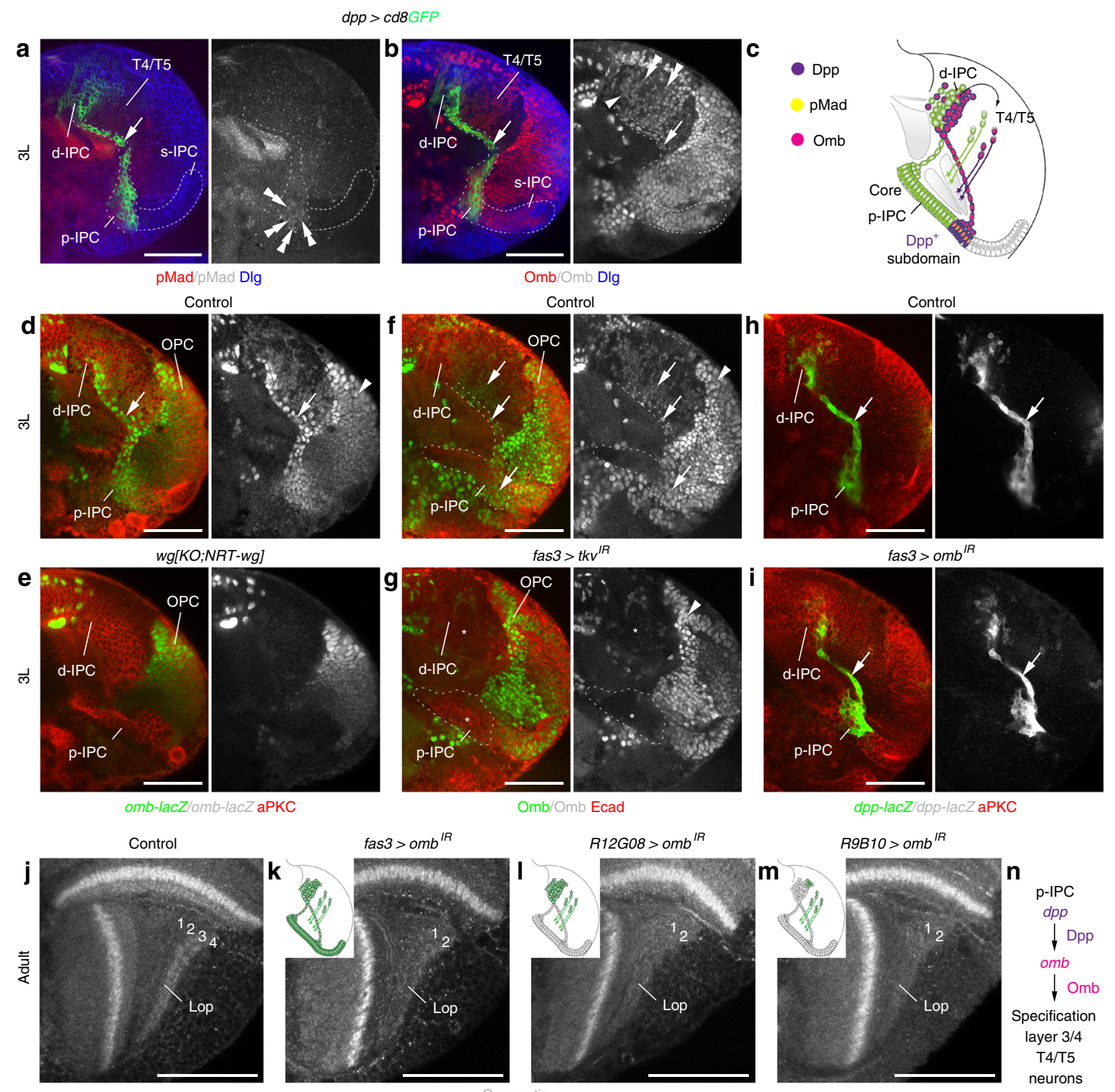

Fig. 7 Omb mediates Dpp-dependent specification of T4/T5 neurons for layers 3/4. a Phosphorylated Mad (pMad, red) in the dpp-Gal4 UAS-cd8GFP (green) expression domain was found in p-IPC NE cells (double arrowheads), but not in progenitors (arrow), d-IPC Nbs/GMCs, or T4/T5 neurons. b Omb (red) was maintained in the dpp-Gal4 UAS-cd8GFP (green) expression domain in progenitors (arrow), d-IPC Nbs/GMCs (arrowhead), and T4/T5 neuron subsets (double arrowheads). c Schematic illustrating pMad and Omb distribution within the Dpp expression domain. d, e Unlike in controls (d), omb ${ }^{P 1}$-lacZ (green) was absent from the IPC (arrow) in wg $\{K O ; N R T$-wg $\}$ flies (e). OPC expression (arrowhead) was not affected. $\mathbf{f}, \mathbf{g}$ In controls (f), Omb protein (green) was detected in p-IPC subdomains, migratory progenitors, and progeny (arrows). Following fas $3^{N P 1233}$-Gal4-mediated IPC-specific tkv knockdown $(\mathbf{g})$, expression was severely reduced in these cells (asterisks). OPC expression (arrowhead) was not affected. $\mathbf{h}$, $\mathbf{i}$ Indistinguishable from controls (h),

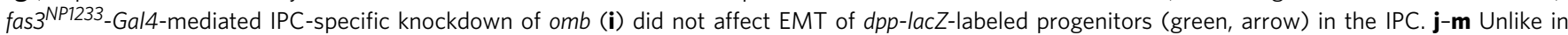
controls (j), omb knockdown in the entire IPC and its progeny using fas $3^{\mathrm{NP1233}}-\mathrm{Gal} 4$ (k), in the d-IPC and postmitotic T4/T5 neurons using R12G08-Gal4 (I), and primarily postmitotic T4/T5 neurons using R9B10-Gal4 ( $\mathbf{m})$ resulted in the absence of Connectin-positive lobula plate layers 3/4. Schematic insets highlight cell type-specificities of Gal4 lines in green. $\mathbf{n}$ Schematics summarizing the role of Dpp in inducing Omb expression to specify layer $3 / 4$ innervating T4/T5 neurons. For genotypes and sample numbers, see Supplementary Table 1. Scale bars, $50 \mu \mathrm{m}$

When Wg is membrane-tethered, the first step of this cascade is disrupted. This defect is not caused by decreased signaling activity of NRT-Wg protein in $w g\{K O ; N R T-w g\}$ flies. First, wild-type Wg signaling activity inside the GPC areas and the adjacent OPC was not affected. Second, in allele switching experiments, ectopic expression of a highly active UAS-NRT-wg transgene in the GPC areas was unable to rescue. By contrast, restoring wild-type $w g$ function in the GPC areas was able to rescue, supporting the notion that $\mathrm{Wg}$ release and spread from the GPC areas are required to induce its own expression in the $\mathrm{s}$-IPC and the $\mathrm{Nb}$ clone.

Although $\mathrm{Wg}$ release is essential, the range of action is likely limited. Wg expression in the s-IPC commences in early 3rd instar larvae, when it is still in close proximity with the GPC. Half of the $w g\{K O ; N R T-w g\}$ flies showed residual $d p p$ expression in one progenitor stream at the 3rd instar larval stage and a $25 \%$ reduction of T4/T5 neurons, correlating with three lobula plate layers in adults. The other half lacked $d p p$-lacZ expression and 
showed a 50\% reduction of T4/T5 neurons correlating with two remaining layers. While this partial phenotypic penetrance is not fully understood, NRT-Wg likely partially substituted for Wg because of the initial close proximity of the GPC areas and the $\mathrm{s}$-IPC and $\mathrm{Nb}$ clone. Occasional residual NRT-Wg expression in the s-IPC argues against an all-or-nothing inductive event and suggests a model, whereby cell-intrinsic signaling thresholds have to be reached. Theoretically, the $d p p$ expression defect in the p-IPC of $w g\{K O ; N R T-w g\}$ flies could reflect the dependence on long-range $\mathrm{Wg}$ from the GPC areas. However, as we have shown, IPC-specific $w g$ knockdown leads to $d p p$ loss in the p-IPC. Propagation of sequential Wnt signaling could explain long-range
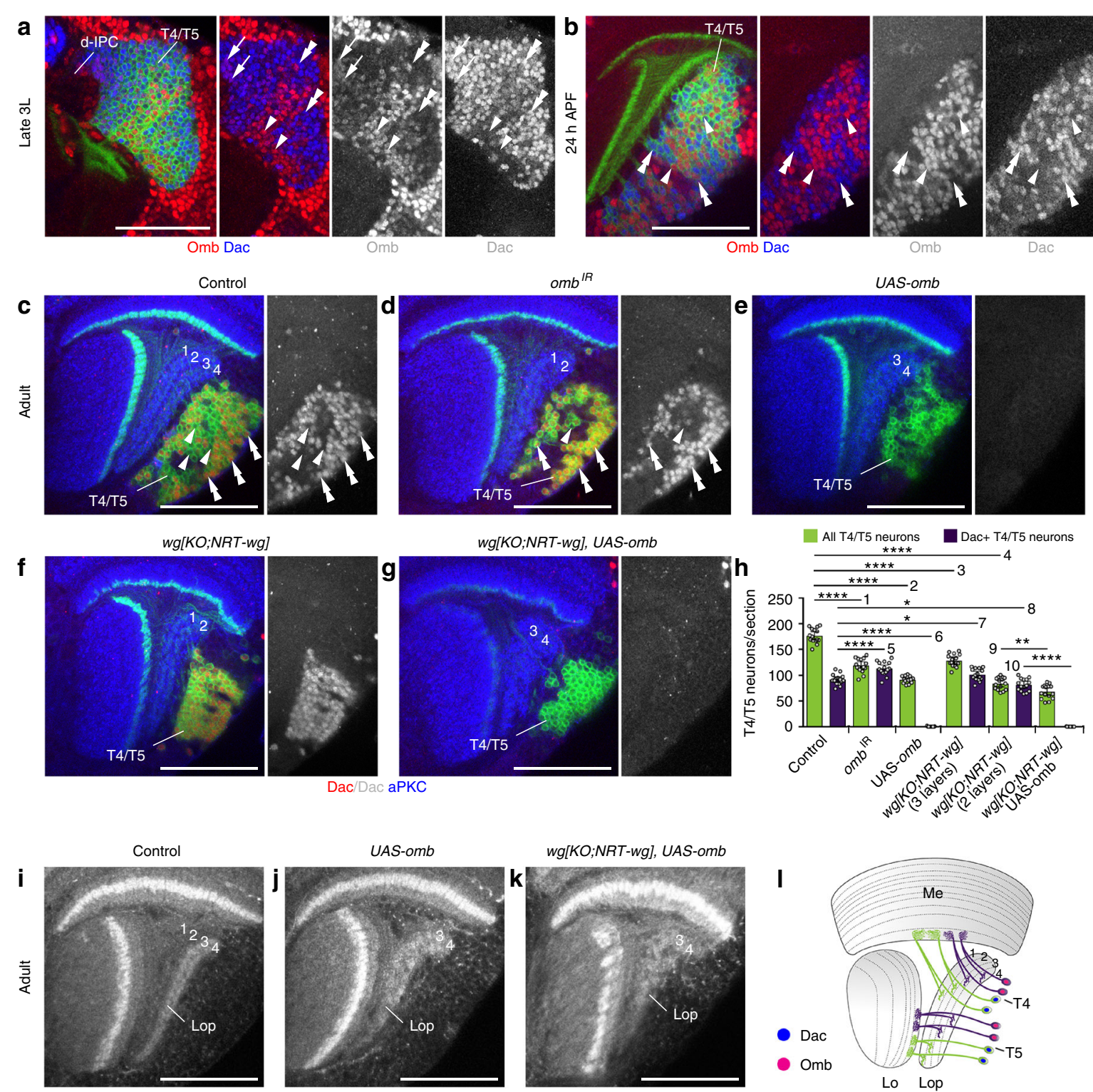

m

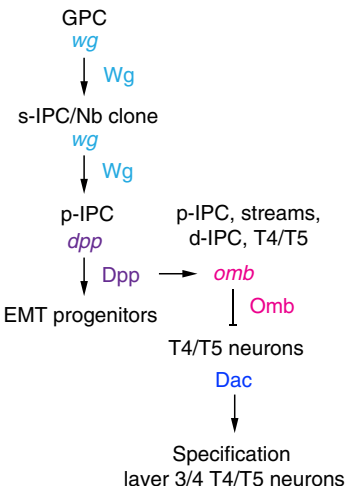

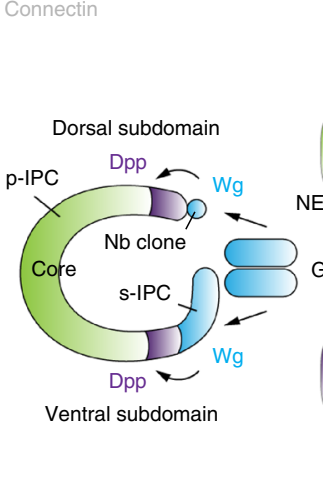

Spatial relay

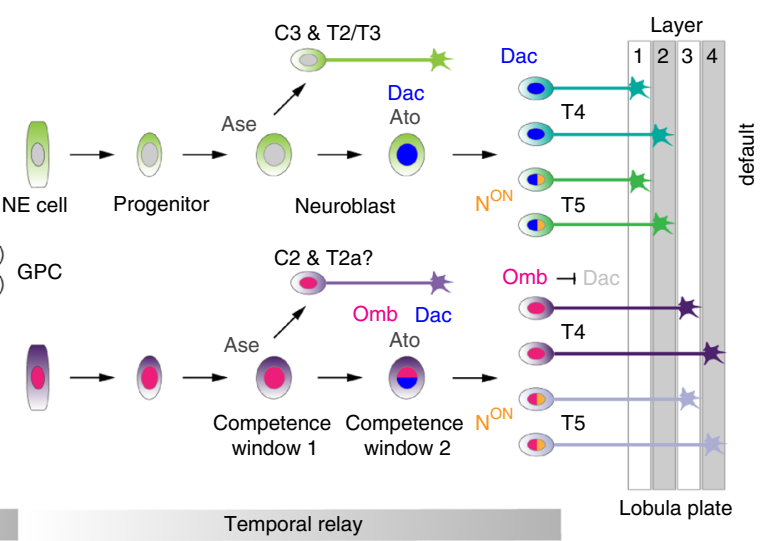


activities $^{38,39}$. Moreover, sequentially acting primary and secondary sources of $\mathrm{Wg}$ have been described in the developing Drosophila eye $\mathrm{e}^{40}$, suggesting that the regulatory mechanism observed in the optic lobe might be employed in several contexts. The different outcomes of early and late allele $w g$ to NRT-wg allele switching indicate that $\mathrm{Wg}$ secretion is required for the induction but not long-term maintenance of $w g$ expression in the s-IPC. The GPC areas become rapidly separated from the s-IPC and Nb clone by compact rows of newly generated neurons. As part of a relay system, diffusible $\mathrm{Wg}$ may therefore be required to bridge distances over a few cell diameters during the initial phase of neurogenesis. The s-IPC in $w g\{K O ; N R T-w g\}$ flies expressed Hth and generated two neuron clusters as in wild-type. Thus, the sole function of $w g$ in the s-IPC is to relay the GPC-derived Wg signal to induce $d p p$ expression in the p-IPC. Since $\mathrm{Wg}$ release is not required in the GPC areas to induce $d p p$ in the adjacent OPC, this secondary $w g$ function in the s-IPC is most likely juxtacrine.

Compared to approximately 80 medulla neuron subtypes derived from the $\mathrm{OPC}^{36}$, the specification of 13 distinct subtypes originating from the p-IPC appears simple. However, the distinct mechanisms employed are surprisingly complex. We previously showed that cross-regulatory interactions between $D$ and tll regulate a $\mathrm{Nb}$ competence switch from generating early-born $\mathrm{C} 2$, C3, T2, T2a, and T3 neurons to eight distinct layer-specific T4/T5 subtypes. Ato and Dac are expressed in the second $\mathrm{Nb}$ competence window and depend on $\mathrm{tl}^{23}$. Our functional studies showed that dac mutant T4/T5 neurons adopted early-born T2/T3 neuron-like morphologies. Similarly, ato mutant T4/T5 neurons displayed neurite connectivity defects ${ }^{41}$. Notably, simultaneous knockdown of $d a c$ and ato resulted in the absence of T4/T5 neurons, demonstrating that both are required together for the ability of d-IPC Nbs to produce new neuron subtypes in the second competence window.

Dac is initially expressed in all T4/T5 neurons but only maintained in layer $1 / 2$ innervating subtypes. This suggests that an essential step for the specification of layer 3/4 innervating neurons is the downregulation of Dac and the suppression of the T4/T5 default neuron fate, i.e., layer $1 / 2$ identity. Although the mode of this inhibitory mechanism depends on the outcome of the $\mathrm{Nb}$-specific switching mechanism in the d-IPC, it is already primed in p-IPC NE cells. Thus, layer-specificity and therefore motion-directionality are determined early in the NE precursors of T4/T5 neurons. Molecularly, it involves the Omb-mediated relay of Dpp-signaling-dependent NE cell patterning information across intermediate cell states to postmitotic T4/T5 neurons resulting in the repression of Dac. In contrast to the $\mathrm{OPC}^{36}$, we found no link between NE patterning in the p-IPC and Notch-dependent differential apoptosis of region-specific
T4/T5 subtypes. Instead, Notch controls the choice between T4 and T5 identity, likely during the second competence window, indicating that the distinction between layer $1 / 2$ and $3 / 4$ fates precedes T4 and T5 neuron specification.

The mechanisms controlling the maintenance of omb expression, and Omb-mediated downregulation of Dac are unclear. Hypotheses regarding the latter have to be reconciled with the fact that $d a c$, together with ato, is required for the formation of all T4/T5 neurons and hence is expressed in all d-IPC Nbs during the second competence window. Omb and Dac are initially coexpressed in Nbs and young T4/T5 neurons, suggesting that Omb does not directly repress dac transcription. Yet, expression of the $d a c^{p 7 d 23}$ enhancer trap Gal4 line showed that dac is only transcribed in layer $1 / 2$ neurons in adults. A possible scenario is that Omb could break Dac autoregulation by triggering degradation of Dac. Since T-box genes can act as transcriptional activators and repressors $^{42}$ and their effects are influenced by various cofactors ${ }^{43}$, future studies will need to explore the molecular details underlying Omb-mediated repression of Dac. It will also be important to determine whether layer $3 / 4$ specification is mediated solely by Dac downregulation, or whether omb has additional instructive roles.

Consistent with the observation that $\mathrm{C} 2$ and $\mathrm{C} 3$ neurons have distinct developmental origins ${ }^{44}$, we found that Nbs derived from the Dpp-expression domain produce $\mathrm{C} 2$ and possibly $\mathrm{T} 2 \mathrm{a}$ neurons during the first $\mathrm{Nb}$ competence window, while the core p-IPC generates C3, T2, and T3 neurons. dac mutant T4/T5 neurons adopt T2/T3-like morphologies suggesting that this is the default neuron fate in this neuron group. While Omb is maintained in C\&T neurons derived from the Dpp-expression domain, Dac is not expressed, suggesting that Omb interacts with other molecular determinants in these neurons. While we did not explore how layer 1 and 2 neurons or layer 3 and 4 neurons become distinct from each other because of the lack of specific markers, our data suggest a possible contribution of Ato/Dac and Notch signaling, as these are active within the d-IPC. Findings in a concurrent study of Pinto-Teixeira et al. ${ }^{45}$ align with our data concerning the role of Dpp and Notch signaling. Furthermore, a second study of Mora et al. ${ }^{46}$ reported an additional role for Ato in controlling the transient amplification of d-IPC Nbs by symmetric cell division to ensure that the correct number of T4/T5 neurons is produced. It will be fascinating to identify the transcriptional targets of Notch, Ato/Dac, and Omb that mediate ganglion- and layer-specific targeting of T4/T5 dendrites and axons, respectively. Finally, future behavioral studies of layer 3/4-deficient flies will address to what extent direction selectivity is affected or compensatory mechanisms are in place.

Fig. 8 Omb converts layer $1 / 2$ into layer 3/4 T4/T5 neurons by Dac downregulation. a, b In the 3rd instar larvae (a), Omb (red) and Dac (blue) were initially co-expressed (arrows) in new-born R9B10-Gal4 UAS-cd8GFP (green) labeled T4/T5 neurons close to the d-IPC. In some more mature T4/T5 neurons, that were positive for Omb, Dac expression was low (arrowheads), while in others, Dac expression was high (double arrowheads) and Omb expression was absent. At $24 \mathrm{~h}$ after puparium formation (APF) (b), Dac (double arrowheads) and Omb (arrowheads) show mutually exclusive expression. c-g In controls (c), Dac (red, double arrowheads) was expressed in approximately 50\% of T4/T5 neurons. Arrowheads indicate Dac-negative R9B10-Gal4 UAS-cd8GFP (green) labeled T4/T5 neurons. Dac was maintained in almost all T4/T5 neurons following omb knockdown (d), and downregulated following omb overexpression (e). Dac was expressed in all T4/T5 neurons in wg\{KO;NRT-wg $\}$ flies with two lobula plate layers (f). Ectopic Omb was sufficient to downregulate Dac in these flies ( $\mathbf{g}$ ). $\mathbf{h}$ Quantification of all and Dac-positive T4/T5 neuron numbers following omb manipulations. The scatter plot with bars shows data points and means with $\pm 95 \%$ confidence interval error bars $(n=15$ corresponding to three serial optical sections, 6 - $\mu \mathrm{m}$ apart, from five samples per genotype). Unpaired, two-tailed Student's $t$-test not assuming equal variance: $P=5.57 \times 10^{-12}, P=6.19 \times 10^{-15}, P=1.26 \times 10^{-10}, P=1.98 \times$ $10^{-17}, P=3.60 \times 10^{-5}, P=4.79 \times 10^{-15}, P=0.020, P=0.017, P=0.0015, P=2.42 \times 10^{-13}$. ${ }^{\star} P<0.05 ;{ }^{\star \star} P<0.01 ;{ }^{\star \star \star \star *} P<0.0001$. i-k Unlike in controls (i), R9B10-Gal4-mediated ectopic UAS-omb expression in T4/T5 neurons of wild type (j) or wg $\{K O$;NRT-wg\} (k) flies resulted in ectopic Connectin expression in the lobula plate (Lop). I Schematic illustrating Dac and Omb expression in adults. $\mathbf{m}$ Working model for spatial and temporal relay mechanisms regulating the formation and specification of layer-specific T4/T5 neurons. For genotypes and sample numbers, see Supplementary Table 1. Scale bars, $50 \mu \mathrm{m}$ 
Signaling centers, also called organizers, pattern tissues in a non-autonomous fashion ${ }^{47}$. The vertebrate roof plate and the cortical hem, for instance, both release Wnts and Bmps to pattern $\mathrm{NE}$ cells in the developing dorsal spinal cord and in the surrounding forebrain, respectively ${ }^{47-49}$. In the Drosophila visual system, the GPC areas express $w g$ and pattern the OPC by inducing $d p p$ expression in adjacent dorsal and ventral OPC subdomains ${ }^{26}$. Together with our insights into the function of GPC-derived $w g$ in IPC patterning and neurogenesis, this firmly establishes the GPC areas as local organizers of optic lobe development. At the onset of neurogenesis, $w g$ is first expressed in the GPC areas followed by the s-IPC, explaining the wellestablished delay in neurogenesis between the IPC and $\mathrm{OPC}^{20}$. Wg release from the GPC areas could coordinate the timely onset of neurogenesis in the OPC and IPC to safeguard the alignment of matching partner neurons across several retinotopically organized neuropils. The intercalation of new-born neurons between both neuroepithelia may have driven the need for a relay system using primary and secondary sources of Wg. Wg induces Dpp to subdivide the adjacent OPC and p-IPC NE into distinct regions as basis for generating neuronal diversity. The temporal relay mediated by Omb represents an efficient strategy to pass the memory of spatial NE patterning information by Dpp signaling on to postmitotic neurons generated at a distance. It is thus intricately tuned to the distinct neurogenesis mode of the p-IPC essential for spatially matching birth-order-dependent neurogenesis between the OPC and IPC ${ }^{23}$. Interestingly, the progressive refinement of NE patterning by the induction of secondary signaling centers plays a central role in vertebrate brain development ${ }^{47}$. Furthermore, similar signaling cascades have been recently identified in mammalian optic tissue cultures where sequential Wnt and Bmp signaling induces the expression of the Omb-related T-box transcription factor Tbx 5 to specify dorsal retinal NE cells ${ }^{50}$. Hence, such cascades could represent conserved regulatory modules that are employed repeatedly during invertebrate and vertebrate nervous system development.

\section{Methods}

Genetics. Drosophila melanogaster strains were maintained on standard medium at $25^{\circ} \mathrm{C}$ except for Gal80 ts and RNAi experiments, in which progeny were shifted from $18^{\circ} \mathrm{C}$ or $25^{\circ} \mathrm{C}$ to $29^{\circ} \mathrm{C}$ at specific time points as indicated below; $w^{1118}$ flies were used as controls. If not otherwise indicated, all stocks were obtained from the Bloomington Drosophila Stock Center or the Vienna Drosophila Resource Center and are described in FlyBase. Crosses involved approximately five males and seven virgin females. To avoid overcrowding, parents were transferred to fresh vials every day or every second day. Control and experimental animals of the correct age and genotype were selected irrespective of their gender randomly and independently from several vials. If not otherwise indicated, wandering 3rd instar larvae were analyzed. Detailed genotypes of all experimental samples are listed in Supplementary Tables 1 and 2 .

The following stocks served as reporter lines: (1) fz3-GFP (Flytrap\#G00357) ${ }^{51}$, (2) dpp-lac $Z^{E x e l .2}$, (3) omb $b^{P 1}$-lac $Z^{52}$, (4) notum ${ }^{W R E}$-lacZ (from J. P. Vincent, The Francis Crick Institute, London). The following lines were used as Gal4 drivers: (1) $d a c^{p 7 d 23}-G a l 4 / C y O^{53},(2) d p p^{b l k 1}-G a l 4 / C y O$ (from J.P. Vincent), (3) fas $3^{N P 1233}$ Gal4 (Kyoto Drosophila Stock Center), (4) fkh-Gal4 (from A. Gould, The Francis Crick Institute, London), (5) $h^{113}$-Gal4 (from A. Brand, The Gurdon Institute, Cambridge), (6) R9B10-Gal4, (7) R9H07-Gal4, (8) R12G08-Gal4, (9) R17B05-Gal4, (10) R17C06-Gal4, (11) R34E01-Gal4, (12) R45H05-Gal4, (13) R46E01-Gal4, (14) R67E05-Gal4 ${ }^{54-56}$, (15) wg-Gal4/CyO, (16) wg $\{$ KO;Gal4 $\} / C y O^{30}$, (17) $w g\{K O ; G a l 4\} /$ $C y O$; UAS-HRP-cd8GFP/TM2 $2^{30}$. For presynaptic labeling, UAS-brp-RFP (from S. Sigrist, FU Berlin) was used; for membrane-tethered GFP reporter gene expression, (1) UAS-cd8GFP, (2) UAS-FB1.1B ${ }^{57}$, or (3) UAS-FB1.1C. In UAS-FB1.1C, cd8mCherry and myr/palm (mp)-tethered mTurquoise in $U A S-F B 1.1 B$ were replaced by TagRFP-T and mTurquoise2, respectively (unpublished). For R45H05-Gal4 cell lineage analysis using the $h s$-FLPout approach ${ }^{58}$, act $>y^{+}>$Gal4 UAS-GFP/GlaBc;

UAS-FLP/TM6B flies were crossed with tubP-Gal80 ${ }^{t s}$; R45H05-Gal4 and kept at 18 ${ }^{\circ} \mathrm{C}$ before shifting to $29^{\circ} \mathrm{C}$ at the late $3 \mathrm{rd}$ instar larval stage. For Flybow experiments $^{57,59}$, (1) $h s$ - $m F L P 5^{M H 12} / G l a B c$ flies were crossed with (2) UAS $F B 1.1 B^{260 b}$; R9B10-Gal4 and (3) UAS-FB1.1B $B^{260 b}$; R9H07-Gal4. Progeny were heat shocked for $40-60 \mathrm{~min}$ at $48-72 \mathrm{~h}$ and $72-96 \mathrm{~h}$ or at $24-48 \mathrm{~h}, 48-72 \mathrm{~h}$, and $72-96 \mathrm{~h}$ after egg laying (AEL) in a $37^{\circ} \mathrm{C}$ water bath. Loss-of-function analysis using mosaic analysis with a repressible cell marker (MARCM) ${ }^{60}$ were conducted by crossing $y w$
hs-FLP ${ }^{122}$; tubP-Gal80 ${ }^{L 10}$ FRT40A/CyO; UAS-FB1.1B ${ }^{49 b}$ R9B10-Gal4/TM6 flies with (1) FRT40A, (2) dac $c^{1}$ FRT40A, (3) FRT40A; UAS-brp-RFP, and (4) dac ${ }^{1}$ FRT40A; UAS-brp-RFP ( $d a c^{1}$ from F. Pignoni, SUNY Upstate Medical University). Twenty-four-hour embryo collections were heat shocked for 40-60 min at 48-72 h and $72-96 \mathrm{~h}$ AEL in a $37^{\circ} \mathrm{C}$ water bath. For knockdown experiments using UASRNAi transgenes, the following driver lines were used: (1) yw ey $y^{3.5}$-Gal80;

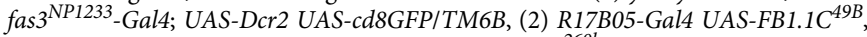
(3) UAS-cd8GFP; R12G08-Gal4, and (4) UAS-FB1.1B ${ }^{260 b}$; R9B10-Gal4. RNAi lines used were: (1) UAS-ato ${ }^{I R}$ TRiP.JF02089, (2) UAS-dac ${ }^{I R K 106040}$, (3) UAS-fz $z^{I R}$ GD43077 (4) UAS-fz $2^{I R}$ KK108998, (5) UAS-omb ${ }^{I R C 1}$ (ref. ${ }^{61}$; from G. Pflugfelder, University of Mainz), (6) UAS-omb $b^{I R ~ K K 100598},(7) U A S-S u(H)^{I R ~ K K 103597},(8) U A S-t k v^{I R} K K 105834$ and (9) UAS-wg $g^{I R}$ GD13351. UAS-Dcr2 was co-expressed in all experiments. Progeny were shifted from $25^{\circ} \mathrm{C}$ to $29^{\circ} \mathrm{C} 24 \mathrm{~h}$ AEL. Exceptions were crosses of ey $y^{3.5}$-Gals0 fas $3^{N P 1233}$-Gal4; UAS-Dcr2 UAS-cd8GFP and UAS-omb ${ }^{I R}$ or UAS-tkv $v^{I R}$ animals, which were kept at $18^{\circ} \mathrm{C}$ before shifting to $29^{\circ} \mathrm{C}$ at the early $3 \mathrm{rd}$ instar larval stage. For UAS-NRT-wg ${ }^{33}$ gain-of-function experiments, $w g\{K O ; G a l 4\} / C y O ; U A S-F L P$ flies were crossed with $w g\left\{K O ; F R T w g^{+}\right.$FRT NRT-wg\} UAS-NRT-wg; tubP-Gal80 ${ }^{\text {ts }}$ flies and kept at $18^{\circ} \mathrm{C}$. While experimental animals were shifted to $29^{\circ} \mathrm{C}$ at the 1 st instar larval stage, control animals were maintained at $18^{\circ} \mathrm{C}$ until the mid $3 \mathrm{rd}$ instar larval stage and then shifted to $29^{\circ} \mathrm{C}$. $w g\left\{K O ; F R T w g^{+}\right.$FRT NRT-wg\} flies are described in ref. ${ }^{30}$. For GPC-specific $w g\left\{K O ; F R T\right.$ NRT-wg FRT $\left.w g^{+}\right\}$allele switching rescue experiments ${ }^{30}, w g\left\{K O ; F R T\right.$ NRT-wg FRT $\left.w g^{+}\right\} / G l a B c$; UAS-FLP flies were crossed with (1) $w g\{K O ; N R T-w g\} / G l a B c^{30}$ and (2) $w g\{K O ; N R T-w g\} /$ GlaBc; R46E01-Gal4 and maintained at $25^{\circ} \mathrm{C}$. For gain-of-function experiments, $U A S-c d 8-G F P ; h^{133}$-Gal4 was crossed with UAS-arm ${ }^{S 10}$; dpp-lac $Z^{\text {Exel.2 }}$ (ref. ${ }^{62}$ ), $U A S-F B 1.1 B^{260 b} ; R 9 B 10-G a l 4$ was crossed with UAS-N ${ }^{\text {intra }}$ (from L. Tsuda, NCGG, $\mathrm{Obu}$ ), and (1) UAS-FB1.1B ${ }^{260 b}$; R9B10-Gal4 or (2) $w g\{K O ; N R T-w g\} / G l a B c ; R 9 B 10-$ Gal4 UAS-FB1.1B ${ }^{49 b}$ were crossed with UAS-omb ( $\$ 2-1$, ref. ${ }^{63}$; from G. Pflugfelder) and $w g\{K O ; N R T-w g\} / G l a B c ; U A S-o m b$.

Immunolabeling and imaging. Brains were dissected in phosphate-buffered saline (PBS), fixed for $1 \mathrm{~h}$ at $20-24^{\circ} \mathrm{C}$ in $2 \%$ paraformaldehyde (wt/vol) in $0.05 \mathrm{M}$ sodium phosphate buffer ( $\mathrm{pH} 7.4$ ) containing $0.1 \mathrm{M} \mathrm{L-lysine} \mathrm{(Sigma-Aldrich)} \mathrm{and} \mathrm{washed}$ in PBS containing $0.5 \%$ Triton X-100 (Sigma-Aldrich). Primary and secondary antibodies were diluted in 10\% Normal Goat Serum (NGS) and PBT. The following primary antibodies were used: rabbit antibody to Ase (1:5000, from Y. N. Jan, HHMI, San Francisco $\left.{ }^{64}\right)$, rabbit antibody to Ato (1:5000, from Y. N. Jan $\left.{ }^{65}\right)$, mouse antibody to Brp (nc82, 1:10, Developmental Studies Hybridoma Bank [DSHB]), mouse antibody to Connectin (C1.427, 1:40, DSHB as marker for lobula plate layers $\left.3 / 4^{66}\right)$, guinea pig antibody to $\mathrm{D}\left(1: 200\right.$, from A. Gould $\left.{ }^{67}\right)$, mouse antibody to Dac (mAbdac2-3, 1:50, DSHB), rabbit antibody to Dcp1 (\#9578, 1:200, Cell Signaling Technologies), mouse antibody to Dlg (4F3, 1:50, DSHB), guinea pig antibody to Dpn (1:500, from J. Skeath, Washington University, St. Louis $\left.{ }^{68}\right)$, rat antibody to E-cad (DCAD2, 1:2, DSHB), mouse antibody to Fas3 (7G10, 1:5, DSHB), chicken, mouse, and rabbit antibodies to $\beta$-galactosidase (\#ab9361, 1:500, Abcam; \#Z3783, 1:300, Promega; \#559762, 1:12,000, Cappel), rabbit antibody to GFP (\#A6455, 1:1000, Molecular Probes), rabbit antibody to Hth (1:100, from R Mann, Columbia University, New York $\left.{ }^{69}\right)$, rabbit antibody to Omb (1:400, G. Pflugfelder, University of Mainz/J.P. Vincent ${ }^{63}$ ), rabbit antibody to aPKC $\varsigma$ (sc-216, 1:100, Santa Cruz Biotechnologies), rabbit antibody to pSmad3 (pS423/425 \#1880-S, 1:2, Epitomics), rabbit antibody to Tll (812, 1:20, J. Reinitz Segmentation Antibodies $^{70}$ ), guinea pig antibody to Toy $(1.170,1: 200$, from U. Walldorf, University of Homburg), and mouse antibody to Wg (4D4, 1:20, DSHB). For immunofluorescence labeling, samples were incubated for $2.5 \mathrm{~h}$ at $20-24^{\circ} \mathrm{C}$ in goat $\mathrm{F}\left(\mathrm{ab}^{\prime}\right)_{2}$ fragments coupled to FITC/DyLight 488, Cy3, or Alexa Fluor 647 (1:400; Jackson ImmunoResearch Laboratories): antibody to guinea pig (Cy3: \#106-166003; Alexa Fluor 647: \#106-606-003), antibody to mouse (DyLight488: \#115-486003; Cy3: \#115-166-003; Alexa Fluor 647: \#115-606-003), antibody to rabbit (FITC \#111-096-003; Cy3: \#111-166-003; Alexa Fluor 647: \#111-606-003), antibody to rat (Cy3: \#112-166-003). Furthermore, goat antibody to chicken IgY (H+L) (Alexa Fluor 555, Molecular Probes, \#A21437, 1:400) was used. Images were collected with a Leica TCS SP5 II laser scanning confocal microscope and processed using Adobe Photoshop and Fiji software programs.

Quantifications and statistics. Statistical details of all experiments are reported in the figures and figure legends. To quantify T4/T5 neuron numbers, adult optic lobes were imaged in horizontal orientations and cell numbers were collected from three serial optical sections $(6-\mu \mathrm{m}$ distance) in five samples $(n=15)$ at the center of the optic lobe. Sample numbers and genotypes for all experiments are provided in Supplementary Tables 1 and 2. If not otherwise indicated, the penetrance of observed phenotypes was $100 \%$ for examined samples. Sample sizes were not predetermined by statistical calculations, but were based on the standard of the field. In a pool of control or experimental animals, specimens of the correct stage and genotype were selected randomly and independently from different vials. Data acquisition and analysis were not performed blinded but relied on samples with identified genotypes that were not limited in repeatability. The calculations of $95 \%$ confidence interval error bars and unpaired two-tailed Student's $t$-test $P$ values were performed using Microsoft Excel software [Confidence.T and T.Test (type 3, not assuming equal variance)]. Prism 7 GraphPad was used to perform ShapiroWilk and D'Agostino-Pearson omnibus normality tests and data met the 
assumption of normality in one or both tests. Quantifications are presented as scatter plots and bar graphs with means $\pm 95 \%$ confidence interval error bars. ${ }^{\star} P<0.05 ;{ }^{*} P<0.01 ;{ }^{* *} P<0.001,{ }^{* * * *} P<0.0001$.

Data availability. Image data sets generated and analyzed in this study are available from the corresponding author upon reasonable request. A source data file for quantifications shown in Figs. $1 \mathrm{~h}$ and $8 \mathrm{~h}$ is provided with this manuscript (Supplementary Data 1).

Received: 23 January 2018 Accepted: 4 May 2018

Published online: 12 June 2018

\section{References}

1. Clark, D. A. \& Demb, J. B. Parallel computations in insect and mammalian visual motion processing. Curr. Biol. 26, R1062-R1072 (2016).

2. Mauss, A. S., Vlasits, A., Borst, A. \& Feller, M. Visual circuits for direction selectivity. Annu. Rev. Neurosci. 40, 211-230 (2017).

3. Gabriel, J. P., Trivedi, C. A., Maurer, C. M., Ryu, S. \& Bollmann, J. H. Layerspecific targeting of direction-selective neurons in the zebrafish optic tectum. Neuron 76, 1147-1160 (2012).

4. Robles, E., Filosa, A. \& Baier, H. Precise lamination of retinal axons generates multiple parallel input pathways in the tectum. J. Neurosci. 33, 5027-5039 (2013).

5. Buchner, E., Buchner, S. \& Bulthoff, I. Deoxyglucose mapping of nervous activity induced in Drosophila brain by visual movement. I. Wildtype. J. Comp. Physiol. A 155, 471-483 (1984).

6. Maisak, M. S. et al. A directional tuning map of Drosophila elementary motion detectors. Nature 500, 212-216 (2013).

7. Huberman, A. D. et al. Genetic identification of an On-Off direction-selective retinal ganglion cell subtype reveals a layer-specific subcortical map of posterior motion. Neuron 62, 327-334 (2009).

8. Kay, J. N. et al. Retinal ganglion cells with distinct directional preferences differ in molecular identity, structure, and central projections. J. Neurosci. 31, 7753-7762 (2011).

9. Hadjieconomou, D., Timofeev, K. \& Salecker, I. A step-by-step guide to visual circuit assembly in Drosophila. Curr. Opin. Neurobiol. 21, 76-84 (2011)

10. Fisher, Y. E., Silies, M. \& Clandinin, T. R. Orientation selectivity sharpens motion detection in Drosophila. Neuron 88, 390-402 (2015).

11. Mauss, A. S., Meier, M., Serbe, E. \& Borst, A. Optogenetic and pharmacologic dissection of feedforward inhibition in Drosophila motion vision. J. Neurosci. 34, 2254-2263 (2014).

12. Fischbach, K. F. \& Dittrich, A. P. M. The optic lobe of Drosophila melanogaster. I. A Golgi analysis of wild-type structure. Cell Tissue Res. 258, 441-475 (1989).

13. Silies, M., Gohl, D. M. \& Clandinin, T. R. Motion-detecting circuits in flies: coming into view. Annu. Rev. Neurosci. 37, 307-327 (2014).

14. Haag, J., Arenz, A., Serbe, E., Gabbiani, F. \& Borst, A. Complementary mechanisms create direction selectivity in the fly. eLife 5, e17421 (2016).

15. Leong, J. C., Esch, J. J., Poole, B., Ganguli, S. \& Clandinin, T. R. Direction selectivity in Drosophila emerges from preferred-direction enhancement and null-direction suppression. J. Neurosci. 36, 8078-8092 (2016).

16. Strother, J. A. et al. The emergence of directional selectivity in the visual motion pathway of Drosophila. Neuron 94, 168-182 e110 (2017).

17. Takemura, S. Y. et al. A visual motion detection circuit suggested by Drosophila connectomics. Nature 500, 175-181 (2013)

18. Takemura, S. Y. et al. The comprehensive connectome of a neural substrate for 'ON' motion detection in Drosophila. eLife 6, e24394 (2017).

19. Apitz, H. \& Salecker, I. A challenge of numbers and diversity: neurogenesis in the Drosophila optic lobe. J. Neurogenet. 28, 233-249 (2014).

20. Hofbauer, A. \& Campos-Ortega, J. A. Proliferation and and early differentiation of the optic lobes in Drosophila melanogaster. Roux Arch. Dev. Biol. 198, 264-274 (1990).

21. Green, P., Hartenstein, A. Y. \& Hartenstein, V. The embryonic development of the Drosophila visual system. Cell Tissue Res. 273, 583-598 (1993).

22. Egger, B., Boone, J. Q., Stevens, N. R., Brand, A. H. \& Doe, C. Q. Regulation of spindle orientation and neural stem cell fate in the Drosophila optic lobe. Neural Dev. 2, 1 (2007).

23. Apitz, H. \& Salecker, I. A region-specific neurogenesis mode requires migratory progenitors in the Drosophila visual system. Nat. Neurosci. 18, 46-55 (2015).

24. Yasugi, T., Umetsu, D., Murakami, S., Sato, M. \& Tabata, T. Drosophila optic lobe neuroblasts triggered by a wave of proneural gene expression that is negatively regulated by JAK/STAT. Development 135, 1471-1480 (2008).

25. Bertet, C. et al. Temporal patterning of neuroblasts controls Notch-mediated cell survival through regulation of Hid or Reaper. Cell 158, 1173-1186 (2014).
26. Kaphingst, K. \& Kunes, S. Pattern formation in the visual centers of the Drosophila brain: wingless acts via decapentaplegic to specify the dorsoventral axis. Cell 78, 437-448 (1994).

27. Apitz, H. \& Salecker, I. Retinal determination genes coordinate neuroepithelial specification and neurogenesis modes in the Drosophila optic lobe. Development 143, 2431-2442 (2016).

28. Petrovic, M. \& Hummel, T. Temporal identity in axonal target layer recognition. Nature 456, 800-803 (2008).

29. Kulkarni, A., Ertekin, D., Lee, C. H. \& Hummel, T. Birth order dependent growth cone segregation determines synaptic layer identity in the Drosophila visual system. eLife 5, e13715 (2016).

30. Alexandre, C., Baena-Lopez, A. \& Vincent, J. P. Patterning and growth control by membrane-tethered Wingless. Nature 505, 180-185 (2014).

31. Hayden, M. A., Akong, K. \& Peifer, M. Novel roles for APC family member and Wingless/Wnt signaling during Drosophila brain development. Dev. Biol. 305, 358-376 (2007).

32. Suzuki, T., Trush, O., Yasugi, T., Takayama, R. \& Sato, M. Wnt signaling specifies anteroposterior progenitor zone identity in the Drosophila visual center. J. Neurosci. 36, 6503-6513 (2016).

33. Zecca, M., Basler, K. \& Struhl, G. Direct and long-range action of a wingless morphogen gradient. Cell 87, 833-844 (1996).

34. Hamaguchi, T., Yabe, S., Uchiyama, H. \& Murakami, R. Drosophila Tbx6 related gene, Dorsocross, mediates high levels of Dpp and Scw signal required for the development of amnioserosa and wing disc primordium. Dev. Biol. 265, 355-368 (2004).

35. Li, X. et al. Temporal patterning of Drosophila medulla neuroblasts controls neural fates. Nature 498, 456-462 (2013).

36. Erclik, T. et al. Integration of temporal and spatial patterning generates neural diversity. Nature 541, 365-370 (2017).

37. Pflugfelder, G. O. et al. The lethal(1)optomotor-blind gene of Drosophila melanogaster is a major organizer of optic lobe development: isolation and characterization of the gene. Proc. Natl Acad. Sci. USA 89, 1199-1203 (1992)

38. Loh, K. M., van Amerongen, R. \& Nusse, R. Generating cellular diversity and spatial form: Wnt signaling and the evolution of multicellular animals. Dev. Cell 38, 643-655 (2016)

39. Nusse, R. \& Clevers, H. Wnt/beta-catenin signaling, disease, and emerging therapeutic modalities. Cell 169, 985-999 (2017).

40. Kumar, S. R., Patel, H. \& Tomlinson, A. Wingless mediated apoptosis: How cone cells direct the death of peripheral ommatidia in the developing Drosophila eye. Dev. Biol. 407, 183-194 (2015).

41. Oliva, C. et al. Proper connectivity of Drosophila motion detector neurons requires Atonal function in progenitor cells. Neural Dev. 9, 4 (2014).

42. Mihalas, A. B. \& Hevner, R. F. Control of neuronal development by T-box genes in the brain. Curr. Top. Dev. Biol. 122, 279-312 (2017).

43. Papaioannou, V. E. The T-box gene family: emerging roles in development, stem cells and cancer. Development 141, 3819-3833 (2014).

44. Nern, A., Pfeiffer, B. D. \& Rubin, G. M. Optimized tools for multicolor stochastic labeling reveal diverse stereotyped cell arrangements in the fly visual system. Proc. Natl Acad. Sci. USA 112, E2967-E2976 (2015).

45. Pinto-Teixeira, F. et al. Development of concurrent retinotopic maps in the fly motion detection circuit. Cell 173, 485-498 e411 (2018).

46. Mora, N. et al. A temporal transcriptional switch governs stem cell division, neuronal numbers, and maintenance of differentiation. Dev. Cell 45, 53-66 e55 (2018).

47. Kiecker, C. \& Lumsden, A. The role of organizers in patterning the nervous system. Annu. Rev. Neurosci. 35, 347-367 (2012).

48. Hoch, R. V., Rubenstein, J. L. \& Pleasure, S. Genes and signaling events that establish regional patterning of the mammalian forebrain. Semin. Cell Dev. Biol. 20, 378-386 (2009).

49. Caronia-Brown, G., Yoshida, M., Gulden, F., Assimacopoulos, S. \& Grove, E. A. The cortical hem regulates the size and patterning of neocortex. Development 141, 2855-2865 (2014).

50. Hasegawa, Y. et al. Emergence of dorsal-ventral polarity in ESC-derived retinal tissue. Development 143, 3895-3906 (2016).

51. Kelso, R. J. et al. Flytrap, a database documenting a GFP protein-trap insertion screen in Drosophila melanogaster. Nucleic Acids Res. 32, D418-D420 (2004).

52. Tsai, S. F. et al. Gypsy retrotransposon as a tool for the in vivo analysis of the regulatory region of the optomotor-blind gene in Drosophila. Proc. Natl Acad. Sci. USA 94, 3837-3841 (1997).

53. Heanue, T. A. et al. Synergistic regulation of vertebrate muscle development by Dach2, Eya2, and Six1, homologs of genes required for Drosophila eye formation. Genes Dev. 13, 3231-3243 (1999).

54. Pfeiffer, B. D. et al. Tools for neuroanatomy and neurogenetics in Drosophila Proc. Natl Acad. Sci. USA 105, 9715-9720 (2008)

55. Jenett, A. et al. A GAL4-driver line resource for Drosophila neurobiology. Cell Rep. 2, 991-1001 (2012)

56. $\mathrm{Li}, \mathrm{H}$. H. et al. A GAL4 driver resource for developmental and behavioral studies on the larval CNS of Drosophila. Cell Rep. 8, 897-908 (2014). 
57. Shimosako, N., Hadjieconomou, D. \& Salecker, I. Flybow to dissect circuit assembly in the Drosophila brain. Methods Mol. Biol. 1082, 57-69 (2014).

58. Ito, K., Awano, W., Suzuki, K., Hiromi, Y. \& Yamamoto, D. The Drosophila mushroom body is a quadruple structure of clonal units each of which contains a virtually identical set of neurones and glial cells. Development 124, 761-771 (1997).

59. Hadjieconomou, D. et al. Flybow: genetic multicolor cell labeling for neural circuit analysis in Drosophila melanogaster. Nat. Methods 8, 260-266 (2011).

60. Lee, T. \& Luo, L. Mosaic analysis with a repressible cell marker for studies of gene function in neuronal morphogenesis. Neuron 22, 451-461 (1999).

61. Shen, J., Dorner, C., Bahlo, A. \& Pflugfelder, G. O. Optomotor-blind suppresses instability at the A/P compartment boundary of the Drosophila wing. Mech. Dev. 125, 233-246 (2008).

62. Pai, L. M., Orsulic, S., Bejsovec, A. \& Peifer, M. Negative regulation of Armadillo, a Wingless effector in Drosophila. Development 124, 2255-2266 (1997).

63. Grimm, S. \& Pflugfelder, G. O. Control of the gene optomotor-blind in Drosophila wing development by decapentaplegic and wingless. Science 271, 1601-1604 (1996).

64. Brand, M., Jarman, A. P., Jan, L. Y. \& Jan, Y. N. asense is a Drosophila neural precursor gene and is capable of initiating sense organ formation. Development 119, 1-17 (1993).

65. Jarman, A. P., Sun, Y., Jan, L. Y. \& Jan, Y. N. Role of the proneural gene, atonal, in formation of Drosophila chordotonal organs and photoreceptors. Development 121, 2019-2030 (1995).

66. Gao, S. et al. The neural substrate of spectral preference in Drosophila. Neuron 60, 328-342 (2008).

67. Russell, S. R., Sanchez-Soriano, N., Wright, C. R. \& Ashburner, M. The Dichaete gene of Drosophila melanogaster encodes a SOX-domain protein required for embryonic segmentation. Development 122, 3669-3676 (1996).

68. Bier, E., Vaessin, H., Younger-Shepherd, S., Jan, L. Y. \& Jan, Y. N. Deadpan, an essential pan-neural gene in Drosophila, encodes a helix-loop-helix protein similar to the hairy gene product. Genes Dev. 6, 2137-2151 (1992).

69. Noro, B., Culi, J., McKay, D. J., Zhang, W. \& Mann, R. S. Distinct functions of homeodomain-containing and homeodomain-less isoforms encoded by homothorax. Genes Dev. 20, 1636-1650 (2006).

70. Kosman, D., Small, S. \& Reinitz, J. Rapid preparation of a panel of polyclonal antibodies to Drosophila segmentation proteins. Dev. Genes Evol. 208, 290-294 (1998).

\section{Acknowledgements}

We are grateful to C. Alexandre, A. Baena-Lopez, and J. P. Vincent (The Francis Crick Institute, London) for all Wg reagents and their invaluable advice for this study. We thank A. Brand (The Gurdon Institute, Cambridge), A. Gould (The Francis Crick Institute), Y. N. Jan (HHMI, San Francisco), R. Mann (Columbia University, New York),
G. Pflugfelder (University of Mainz), F. Pignoni (SUNY Upstate Medical University), S. Sigrist (FU Berlin), J. Skeath (Washington University, St. Louis), L. Tsuda (NCGG, Obu), U. Walldorf (University of Homburg), the Bloomington Drosophila Stock Center, the Drosophila Genomics Resource Center, the Vienna Drosophila Resource Center, and the Developmental Studies Hybridoma Bank for fly strains and antibodies. We thank A. Yuen and W. Wang for help with Flybow and MARCM experiments. We thank H. Pynor for advice on the 3D drawing of the larval optic lobe. We thank J. P. Vincent and C. Alexandre, as well as A. Avola, C. de Miguel Vijandi, E. L. Powell, and R. Kaschula (The Francis Crick Institute, London) for critical reading of the manuscript. This work was supported by the Francis Crick Institute which receives its core funding from Cancer Research UK (FC001151), the UK Medical Research Council (FC001151) and the Wellcome Trust (FC001151), and by the UK Medical Research Council (U117581332).

\section{Author contributions}

H.A. and I.S. conceived and designed the study. H.A. performed the experiments and analyzed the data. H.A. and I.S. prepared the manuscript.

\section{Additional information}

Supplementary Information accompanies this paper at https://doi.org/10.1038/s41467018-04592-z.

Competing interests: The authors declare no competing interests.

Reprints and permission information is available online at http://npg.nature.com/ reprintsandpermissions/

Publisher's note: Springer Nature remains neutral with regard to jurisdictional claims in published maps and institutional affiliations.

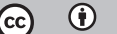

Open Access This article is licensed under a Creative Commons Attribution 4.0 International License, which permits use, sharing, adaptation, distribution and reproduction in any medium or format, as long as you give appropriate credit to the original author(s) and the source, provide a link to the Creative Commons license, and indicate if changes were made. The images or other third party material in this article are included in the article's Creative Commons license, unless indicated otherwise in a credit line to the material. If material is not included in the article's Creative Commons license and your intended use is not permitted by statutory regulation or exceeds the permitted use, you will need to obtain permission directly from the copyright holder. To view a copy of this license, visit http://creativecommons.org/ licenses/by/4.0/.

(c) The Author(s) 2018 